\title{
Efficient Cu-Catalyzed Asymmetric Conjugate Additions of Alkylzinc Reagents to Acyclic Nitroalkenes
}

\author{
Dawn M. Mampreian and Amir H. Hoveyda* \\ Department of Chemistry, Merkert Chemistry Center, Boston College \\ Chestnut Hill, Massachusetts 02467
}

\section{Supporting Information}

General. Infrared (IR) spectra are recorded on a Nicolet 210 spectrophotometer, $v_{\max }$ in $\mathrm{cm}^{-1}$. Bands are characterized as broad (br), strong (s), medium (m) or weak (w). ${ }^{1} \mathrm{H}$ NMR spectra are recorded on a Varian Unity INOVA $400(400 \mathrm{MHz})$ spectrometer. Chemical shifts are reported in ppm from tetramethylsilane with the solvent resonance resulting from incomplete deuteration as the internal standard $\left(\mathrm{CDCl}_{3}: 7.26\right)$. Data are reported as follows: chemical shift, integration, multiplicity $(\mathrm{s}=$ singlet, $\mathrm{d}=$ doublet, $\mathrm{t}=$ triplet, $\mathrm{q}=$ quartet, $\mathrm{br}=$ broad, $\mathrm{m}=$ multiplet), coupling constants, and assignment. ${ }^{13} \mathrm{C}$ NMR spectra are recorded on a Varian Unity INOVA $400(100 \mathrm{MHz})$ spectrometer with complete proton decoupling. Chemical shifts are reported in ppm from tetramethylsilane with the solvent resonance as the internal standard $\left(\mathrm{CDCl}_{3}\right.$ : 77.16). Enantiomer ratios are determined by chiral GLC analysis; (Alltech Associates Chiraldex GTA column, 30m x $0.25 \mathrm{~mm}$ or Supelco Betadex 120 column, $30 \mathrm{~m}$ x $0.25 \mathrm{~mm}$ ) in comparison to authentic racemic materials. Racemic samples are made with a 1:1 mixture of D,D and L,L ligand. Elemental analyses are performed by Robertson Microlit Laboratories (Madison, New Jersey). High resolution mass spectrometry is performed by the University of Illinois Mass Spectrometry Laboratories (Urbana, Illinois) and at the Mass Spectrometry Facility, Boston College (on a Micromass LCT ESI-MS (positive mode)). Optical rotation values are recorded on a Rudolph Research Analytical Autopol IV Polarimeter.

Unless otherwise stated, all reactions are carried out under an inert atmosphere of $\mathrm{N}_{2}$ in flame dried glassware. Solvents are purified under a positive pressure of dry argon by a modified Advanced ChemTech purification system, Toluene is purified through $\mathrm{Cu}$ and alumina columns and $\mathrm{CH}_{2} \mathrm{Cl}_{2}, \mathrm{Et}_{2} \mathrm{O}$ and THF are purified through two alumina colums. Diethylzinc and dimethylzinc are purchased from Aldrich and used without purification. Other alkylzincs are prepared using known methods. ${ }^{1 \mathrm{a}, \mathrm{b}}$ Copper (I) triflate (benzene complex, 2:1) is prepared using known methods, ${ }^{2}$ while copper (I) triflate (toluene complex, 2:1) is purchased form Aldrich and used without purification. trans- $\beta$-Nitrostyrene is purchased from Aldrich and used without further purification. Nitroalkenes $\mathbf{1 b}-\mathbf{1 g}$ are synthesized according to a modified literature procedure. ${ }^{3}$ Nitroalkene $\mathbf{1 h}$ is synthesized according to a literature procedure. ${ }^{4} \mathrm{EDC} \cdot \mathrm{HCl}$,

\footnotetext{
1 a) Knochel, P.; Singer, P. R. Chem. Rev. 1993, 93, 2117-2188 and references cited therein. b) Due to preparation by transmetallation with $\mathrm{Et}_{2} \mathrm{Zn}$, the dialkyl zinc reagents used in entries 3, 4, 7 and 10 in Table 3 are contaminated with mixed $\mathrm{Et}(\mathrm{alkyl}) \mathrm{Zn}$ and $\mathrm{Et}_{2} \mathrm{Zn}$. This leads to ethyl addition byproducts and hence lower yields of the desired product.

2 a) Salomon, R. G.; Kochi, J. K. J. Am. Chem. Soc. 1973, 95, 1889-1897. b) Salomon, R. G.; Kochi, J. K. J. Am. Chem. Soc. 1973, 95, 3300-3310.

${ }^{3}$ Gilman, H.; Blatt, A. H. Organic Syntheses. 1941, 1, 413-415.

4 Duursma, A.; Minnaard, A. J.; Feringa, B. L.; Tetrahedron 2002, 58, 5773-5778.
} 
$\mathrm{HOBt} \cdot \mathrm{H}_{2} \mathrm{O}$, DIC, piperdine, butylamine, diethylamine, dibenzylamine, benzylamine, pyridine, pyrolidine, DMF, Boc- and Fmoc-protected amino acids, and 2(diphenylphosphino)benzaldehyde are purchased from commercial sources (Aldrich and Advanced Chem Tech) and used without further purification.

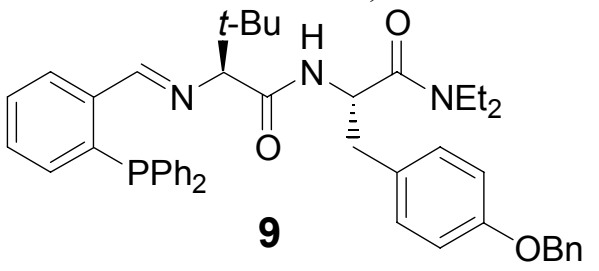

2-(Diphenylphosphino)-L-Tle-L-Tyr(OBn)-NEt 2 (9). To a solution of Boc-L-Tyr(OBn)-OH (2.00 g, $5.38 \mathrm{mmol})$ in $\mathrm{CH}_{2} \mathrm{Cl}_{2}(30 \mathrm{~mL})$ at $22{ }^{\circ} \mathrm{C}$ is added successively $\mathrm{EDC} \cdot \mathrm{HCl}$ (1.03 g, $5.38 \mathrm{mmol})$, HOBt $\cdot \mathrm{H}_{2} \mathrm{O}(0.825 \mathrm{~g}, 5.38 \mathrm{mmol})$, and diethylamine $(1.17 \mathrm{~mL}, 11.30 \mathrm{mmol})$ The resulting solution is allowed to stir at $22{ }^{\circ} \mathrm{C}$ for $4 \mathrm{~h}$. The reaction is quenched by addition of $20 \mathrm{~mL}$ aqueous $10 \mathrm{wt} . \%$ citric acid and the two layers are separated. The organic layer is washed twice with $20 \mathrm{~mL}$ of aqueous $10 \mathrm{wt}$. \% citric acid, $20 \mathrm{~mL}$ of saturated aqueous $\mathrm{NaHCO}_{3}$, and $20 \mathrm{~mL}$ of saturated aqueous $\mathrm{NaCl}$. The organic layer is dried over $\mathrm{MgSO}_{4}$, filtered and concentrated to give a yellow oil. (2.12 g, $\left.4.92 \mathrm{mmol}, 92 \%\right)$.

Trifluoroacetic acid $(6 \mathrm{~mL})$ is added dropwise to Boc-L-Tyr(OBn)NEt $2(2.12 \mathrm{~g}, 4.92 \mathrm{mmol})$ at $0{ }^{\circ} \mathrm{C}$ and allowed to stir for $30 \mathrm{~min}$ at $0{ }^{\circ} \mathrm{C}$. The reaction is quenched with aqueous $6 \mathrm{~N}$ solution of $\mathrm{KOH}$ at $0{ }^{\circ} \mathrm{C}$ until $\mathrm{pH}>10$. The aqueous mixture is washed with $\mathrm{CH}_{2} \mathrm{Cl}_{2}(3 \times 20 \mathrm{~mL})$. The organic layers are combined, dried over $\mathrm{MgSO}_{4}$, filtered, and concentrated to afford a yellow oil (1.45 g, $4.44 \mathrm{mmol}, 90 \%)$.

To a solution of $\mathrm{NH}_{2}-\mathrm{L}-\mathrm{Tyr}(\mathrm{OBn})-\mathrm{NEt}_{2}(1.45 \mathrm{~g}, 4.44 \mathrm{mmol})$ in $\mathrm{CH}_{2} \mathrm{Cl}_{2}(30 \mathrm{~mL})$ at $22{ }^{\circ} \mathrm{C}$ is added successively EDC. $\mathrm{HCl}(0.936 \mathrm{~g}, 4.88 \mathrm{mmol})$, $\mathrm{HOBt} \mathrm{H}_{2} \mathrm{O}(0.748 \mathrm{~g}, 4.88 \mathrm{mmol})$, and BocL-Tle-OH ( $1.03 \mathrm{~g}, 4.44 \mathrm{mmol})$. The resulting solution is allowed to stir at $22{ }^{\circ} \mathrm{C}$ for $4 \mathrm{~h}$ before quenching and extracting as described above to afford Boc-L-Tle-L-Tyr(OBn)-NEt 2 (2.07 g, 3.83 mmol, $86 \%$ ).

Trifluoroacetic acid $(6 \mathrm{~mL})$ is added dropwise to Boc-L-Tle-L-Tyr(OBn)NEt 2 (2.07 $\mathrm{g}, 3.83$ mmol) at $0{ }^{\circ} \mathrm{C}$ and allowed to stir for $30 \mathrm{~min}$ at $0{ }^{\circ} \mathrm{C}$. The reaction is quenched with aqueous $6 \mathrm{~N}$ solution of $\mathrm{KOH}$ at $0{ }^{\circ} \mathrm{C}$ until $\mathrm{pH}>10$. The aqueous solution is washed with $\mathrm{CH}_{2} \mathrm{Cl}_{2}(3 \mathrm{x}$ $20 \mathrm{~mL}$ ). The organic layers are dried over $\mathrm{MgSO}_{4}$, filtered, and concentrated to give a yellow oil $(1.39 \mathrm{~g}, 3.16 \mathrm{mmol}, 83 \%)$.

To a solution of $\mathrm{NH}_{2}$-L-Tle-L-Tyr(OBn)-NEt $2(1.39 \mathrm{~g}, 3.16 \mathrm{mmol})$ in $\mathrm{CH}_{2} \mathrm{Cl}_{2}(20 \mathrm{~mL})$ is added 2-(diphenylphosphino)benzaldehyde (0.917 g, $3.16 \mathrm{mmol})$ and $\mathrm{MgSO}_{4}(0.830 \mathrm{~g}, 4.85$ mmol). The mixture is allowed to stir at $22{ }^{\circ} \mathrm{C}$ for $48 \mathrm{~h}$. After this time, the $\mathrm{MgSO}_{4}$ is filtered and the solvent is evaporated to afford a brownish oil which is purified by silica gel chromatography $\left(2: 1\right.$ hexanes/EtOAc, $\left.\mathrm{R}_{f}=0.10\right)$ to yield an off-white solid $(1.94 \mathrm{~g}, 2.73 \mathrm{mmol}$, 86\%), mp 66-70 ${ }^{\circ} \mathrm{C}$. IR (neat): 3371 (w), 3276 (br), 3062 (w), 2962 (m), 2867 (w), 2357 (w), 1640 (s), 1514 (s), 1464 (m), 1438 (m), 1394 (w), 1375 (w), 1249 (m), 1218 (m), $1180(\mathrm{w}), 1111$ (w), $1086(\mathrm{w}), 1023$ (w), 765 (s), $714(\mathrm{~m}) .{ }^{1} \mathrm{H} \mathrm{NMR}\left(\mathrm{CDCl}_{3}, 400 \mathrm{MHz}\right): \delta 8.62$ (1H, d, J=4.4 $\mathrm{Hz}, \mathrm{N}=\mathrm{CH}), 7.98(1 \mathrm{H}, \mathrm{ddd}, J=7.2,3.6,1.2 \mathrm{~Hz}, \operatorname{ArH}), 7.76(1 \mathrm{H}, \mathrm{d}, J=8.8 \mathrm{~Hz}$, ArH or NH), 7.46-7.26 (15H, m, ArH), 7.22-7.16 (4H, m, ArH), 6.90-6.85 (3H, m, ArH), 5.12-5.06 (1H, m, $\mathrm{NCHCO}), 5.04\left(2 \mathrm{H}, \mathrm{s}, \operatorname{ArCH}_{2} \mathrm{O}\right), 3.46(1 \mathrm{H}, \mathrm{dd}, J=14.0,7.2 \mathrm{~Hz}, \mathrm{CHCHHAr}), 3.36(1 \mathrm{H}, \mathrm{s}$, $\mathrm{NCHCO}$ ), 3.10-2.92 (5H, m, N( $\left.\mathrm{CH}_{2} \mathrm{CH}_{3}\right)_{2}$ and CHCHHAr), 0.98-0.91 (6H, m, $\mathrm{N}\left(\mathrm{CH}_{2} \mathrm{CH}_{3}\right)_{2}$, $0.69(9 \mathrm{H}, \mathrm{s}, t-\mathrm{Bu}) .{ }^{13} \mathrm{C} \mathrm{NMR}\left(\mathrm{CDCl}_{3}, 100 \mathrm{MHz}\right): \delta 170.9,170.7,161.2,161.0,157.8,139.1$, $138.9,137.23,134.7,134.5,134.0,133.9,133.8,130.8,130.7,129.5,129.2,129.1,128.9,128.8$, 128.7, 128.6, 128.1, 127.6, 115.1, 84.2, 70.2, 49.9, 41.9, 40.7, 39.5, 35.2, 27.2, 14.5, 13.0. 
HRMS Calcd. for $\mathrm{C}_{45} \mathrm{H}_{50} \mathrm{~N}_{3} \mathrm{O}_{3} \mathrm{P}\left[\mathrm{M}+\mathrm{H}^{+}\right]$: 712.3668. Found: 712.3688. $[\alpha]_{\mathrm{D}}{ }^{20}+5.66^{\circ}(c=$ 0.353, $\mathrm{CHCl}_{3}$ ).

Representative procedure for the synthesis of nitroalkenes 1b-1g: Nitromethane (1.93 mL, $35.6 \mathrm{mmol})$, 4-chlorobenzaldehyde (2.00 g, $14.2 \mathrm{mmol})$, and methanol $(6.00 \mathrm{~mL}, 0.15$ mmol) are added to a 3-neck round bottomed flask and cooled to $0{ }^{\circ} \mathrm{C}$. Aqueous $1 \mathrm{M} \mathrm{NaOH}$ (35.6 $\mathrm{mL}, 35.6 \mathrm{mmol}$ ) is added by an addition funnel while maintaining the internal reaction temperature between $10-15{ }^{\circ} \mathrm{C}$. Ice water mixture $(25.0 \mathrm{~mL})$ is added and the mixture is allowed to stir at $0{ }^{\circ} \mathrm{C}$ for $15 \mathrm{~min}$. The mixture is slowly added to aqueous $8 \mathrm{M} \mathrm{HCl}(25.0 \mathrm{~mL}, 1.6$ equiv) and allowed to stir until the reaction is confirmed complete by TLC. The mixture is washed with $\mathrm{CH}_{2} \mathrm{Cl}_{2}(3 \times 10 \mathrm{~mL})$. The organic layers are combined and washed with brine, dried over $\mathrm{MgSO}_{4}$, filtered and concentrated to give brown residue. The product is purified by silica gel chromatography (10:1 hexane: $\mathrm{Et}_{2} \mathrm{O}$ to elute product) to afford a yellow crystalline solid (1.18 $\mathrm{g}$, $58 \%$ yield).

Representative procedure for the $\mathrm{Cu}$-catalyzed conjugate addition of dialkyl zinc reagents to acyclic nitroalkene: (CAUTION: $\mathrm{Me}_{2} \mathrm{Zn}$ is pyrophoric! Use extreme caution!) In a glovebox, a $13 \times 100 \mathrm{~mm}$ test tube is charged with $(\mathrm{CuOTf})_{2} \cdot \mathrm{PhH}(0.81 \mathrm{mg}, 1.6 \mu \mathrm{mol})$ and 9 $(2.3 \mathrm{mg}, 3.2 \mu \mathrm{mol})$, sealed with a septum and removed from the glovebox. Toluene $(1.0 \mathrm{~mL})$ is added and the mixture is allowed to stir for $5 \mathrm{~min}$ at $22{ }^{\circ} \mathrm{C}$, then cooled to $-30{ }^{\circ} \mathrm{C}$ in a dry ice/acetone bath. To the mixture, $\mathrm{Me}_{2} \mathrm{Zn}(250 \mu \mathrm{L}$ of a $2.0 \mathrm{M}$ solution in toluene, $0.49 \mathrm{mmol})$ is added dropwise and the resulting solution is allowed to stir at $-30{ }^{\circ} \mathrm{C}$ for $10 \mathrm{~min}$. A solution of trans- $\beta$-nitrostyrene $(24 . \mathrm{mg}, 0.16 \mathrm{mmol}$ ) in $1.0 \mathrm{~mL}$ of toluene is added by syringe and the reaction is allowed to stir at $22{ }^{\circ} \mathrm{C}$. After $24 \mathrm{~h}$, the reaction is quenched at $-30{ }^{\circ} \mathrm{C}$ by the dropwise addition of $5.0 \mathrm{~mL}$ of a saturated aqueous $\mathrm{NH}_{4} \mathrm{Cl}$. The mixture is washed with $\mathrm{Et}_{2} \mathrm{O}(2$ $\mathrm{x} 2.0 \mathrm{~mL}$ ). The combined organic layers are passed through a plug of silica gel with $\mathrm{Et}_{2} \mathrm{O}$. $\mathrm{Et}_{2} \mathrm{O}$ is removed under reduced pressure and the toluene solution is purified by silica gel chromatography ( $100 \%$ pentane to elute toluene followed by $20: 1$ pentane: $\mathrm{Et}_{2} \mathrm{O}$ to elute product) to afford a colorless oil (22 $\mathrm{mg}, 0.13 \mathrm{mmol}, 85 \%$ yield).

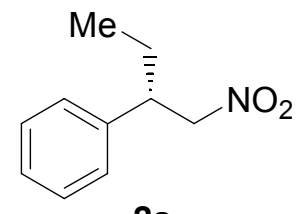

$2 a$

(S)-1-Nitro-2-phenylbutane (2a). IR (neat): 3078 (w), 3035 (m), 2969 (s), 2933 (s), 2879 (m), 1551 (s), 1503 (m), 1461 (m), 1425 (m), 1380 (s), 1208 (w), 1124 (m), 769 (s), 703 (s). ${ }^{1} \mathrm{H} \mathrm{NMR}\left(\mathrm{CDCl}_{3}, 400 \mathrm{MHz}\right): \delta$ 7.36-7.32 (2H, m, ArH), 7.29-7.25 (1H, m, ArH), 7.20-7.18 (2H, m, ArH), 4.61-4.52 $\left(2 \mathrm{H}, \mathrm{m}, \mathrm{CH}_{2} \mathrm{NO}_{2}\right), 3.40-3.33(1 \mathrm{H}, \mathrm{m}, \mathrm{ArCHCH}), 1.82-1.64(1 \mathrm{H}, \mathrm{dqd}, J=$ 14.4, 7.2, $\left.5.6 \mathrm{~Hz}, \mathrm{CHHCH}_{3}\right), 1.82-1.64\left(1 \mathrm{H}, \mathrm{dqd}, J=14.0,9.6,7.2 \mathrm{~Hz}, \mathrm{CHHCH}_{3}\right), 0.85(3 \mathrm{H}$, dd, $\left.J=7.6,7.6 \mathrm{~Hz}, \mathrm{CH}_{2} \mathrm{CH}_{3}\right) .{ }^{13} \mathrm{C} \mathrm{NMR}\left(\mathrm{CDCl}_{3}, 100 \mathrm{MHz}\right): \delta 139.6,129.2,127.9,81.1,46.4,26.5$, 11.5. HRMS Calcd. for $\mathrm{C}_{10} \mathrm{H}_{13} \mathrm{NO}_{2}$ : 179.0946. Found: 179.0945. Anal. Calcd. for $\mathrm{C}_{10} \mathrm{H}_{13} \mathrm{NO}_{2}$ : C, 67.02; H, 7.31; N, 7.82. Found: C, 66.95; H, 6.99; N, 8.03. $[\alpha]_{\mathrm{D}}{ }^{20}-23.1^{\circ}\left(c=0.627, \mathrm{CHCl}_{3}\right)$ for $95 \%$ ee product with $(S)$ stereochemistry. Literature value: $[\alpha]_{\mathrm{D}}{ }^{20}+38.2^{\circ} \quad\left(c=1.10, \mathrm{CHCl}_{3}\right)$ for $97 \%$ ee of $(R)$ product. ${ }^{5}$

The optical purity of 1-nitro-2-phenylbutane is established by chiral GLC analysis ( $\beta$-dex 115 ${ }^{\circ} \mathrm{C}, 15 \mathrm{psi}$ ); chromatograms are illustrated below for a $95 \%$ ee sample:

\footnotetext{
${ }^{5}$ Ohta, H.; Kobayashi, N.; Ozaki, K. J. Org Chem. 1989, 54, 1802-1804.
} 

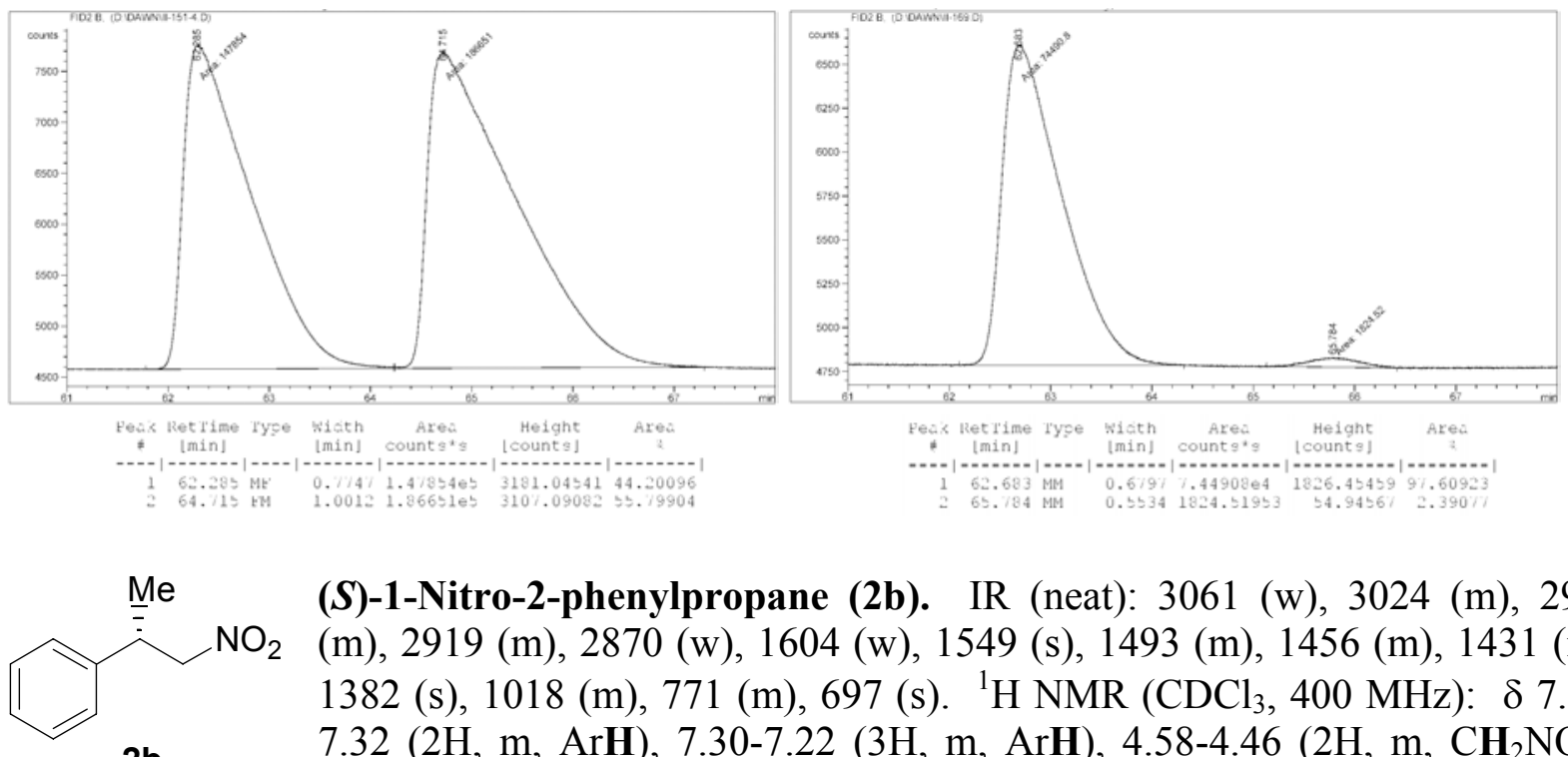

(S)-1-Nitro-2-phenylpropane (2b). IR (neat): 3061 (w), 3024 (m), 2975 (m), 2919 (m), 2870 (w), 1604 (w), 1549 (s), 1493 (m), 1456 (m), 1431 (m), 1382 (s), 1018 (m), $771(\mathrm{~m}), 697$ (s). ${ }^{1} \mathrm{H} \mathrm{NMR}\left(\mathrm{CDCl}_{3}, 400 \mathrm{MHz}\right): \delta 7.37-$ $7.32(2 \mathrm{H}, \mathrm{m}, \mathrm{ArH}), 7.30-7.22(3 \mathrm{H}, \mathrm{m}, \mathrm{ArH}), 4.58-4.46\left(2 \mathrm{H}, \mathrm{m}, \mathrm{CH}_{2} \mathrm{NO}_{2}\right)$, 3.68-3.59 (1H, m, $\operatorname{ArCHCH} 2), 1.39\left(3 \mathrm{H}, \mathrm{d}, J=6.8 \mathrm{~Hz}, \mathrm{CHCH}_{3}\right) .{ }^{13} \mathrm{C} \mathrm{NMR}$ $\left(\mathrm{CDCl}_{3}, 100 \mathrm{MHz}\right): \delta 141.2,129.3,127.9,127.4,82.2,39.0,19.1$. HRMS Calcd. for $\mathrm{C}_{9} \mathrm{H}_{11} \mathrm{NO}_{2}$ : 165.0790. Found: 165.0791. Anal. Calcd. for $\mathrm{C}_{9} \mathrm{H}_{11} \mathrm{NO}_{2}$ : C, 65.44; H, 6.71; N, 8.48. Found: C, 65.15; H, 6.72; N, 8.25. $[\alpha]_{\mathrm{D}}{ }^{20}-48.8^{\circ} \quad\left(c=0.967, \mathrm{CHCl}_{3}\right)$ for $92 \%$ ee product with $(S)$ stereochemistry. Literature value: $[\alpha]_{\mathrm{D}}{ }^{20}+44.3^{\circ}\left(c=3.40, \mathrm{CHCl}_{3}\right)$ for $98 \%$ ee of $(R)$ product. ${ }^{4}$

The optical purity of 1-nitro-2-phenylpropane is established by chiral GLC analysis ( $\beta$-dex, $115^{\circ} \mathrm{C}, 15 \mathrm{psi}$ ); chromatograms are illustrated below for a $92 \%$ ee sample:
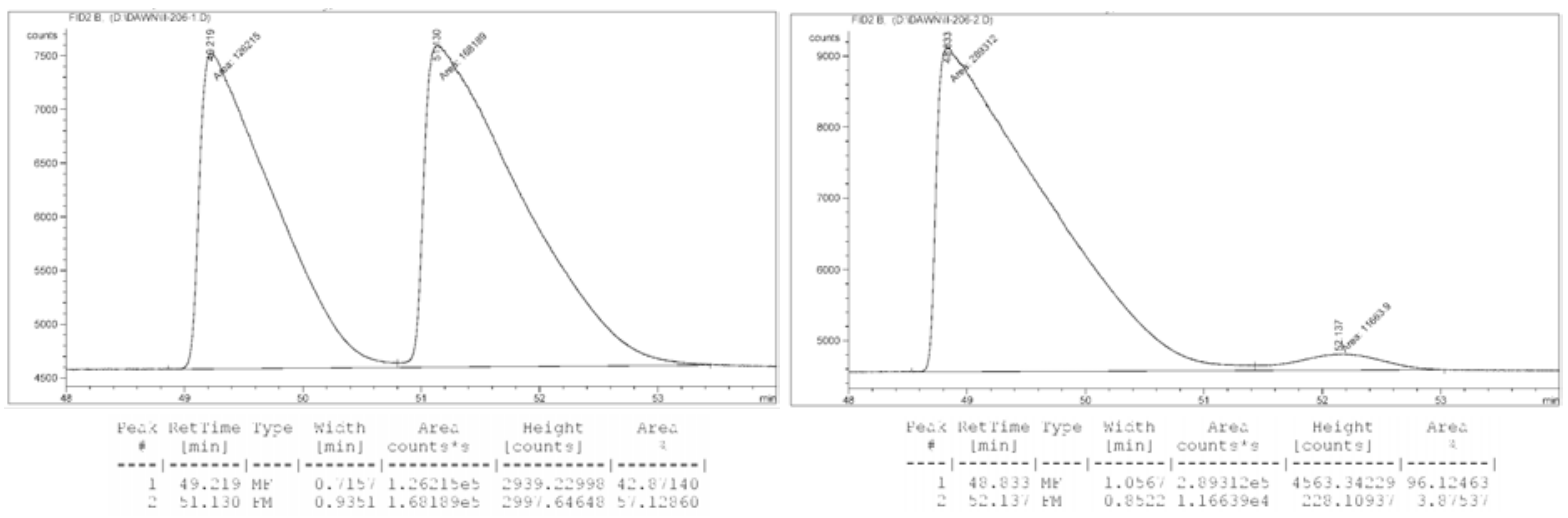

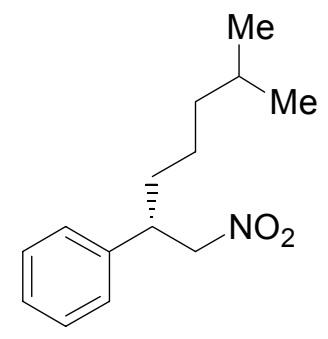

2c

6-Methyl-1-nitro-2-phenylheptane (2c). IR (neat): 3087 (w), 3062 (m), 3031 (m), 2955 (s), 2930 (s), 2867 (s), 1590 (w), 1558 (s), 1495 (m), 1451 (m), 1432 (m), 1382 (s), 1200 (br), 1124 (w), $1080(\mathrm{w}), 1036(\mathrm{w}), 910(\mathrm{w})$, $765(\mathrm{~m}), 697$ (s). ${ }^{1} \mathrm{H} \mathrm{NMR}\left(\mathrm{CDCl}_{3}, 400 \mathrm{MHz}\right): \delta 7.36-7.17(5 \mathrm{H}, \mathrm{m}, \mathrm{ArH})$, 4.59-4.50 (2H, dq, $\left.J=12.0,7.6 \mathrm{~Hz}, \mathrm{CH}_{2} \mathrm{NO}_{2}\right), 3.48-3.41(1 \mathrm{H}$, dddd, $J=7.6$,

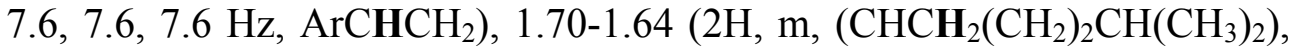
1.50-1.40 (1H, m, $\left.\quad \mathbf{C H}\left(\mathrm{CH}_{3}\right)_{2}\right), \quad 1.23-1.10 \quad(4 \mathrm{H}, \quad \mathrm{m}, \quad$ aliphatic $\left.\mathrm{CHCH}_{2}\left(\mathrm{CH}_{2}\right)_{2} \mathrm{CH}\left(\mathrm{CH}_{3}\right)_{2}\right), 0.80\left(6 \mathrm{H}, \mathrm{t}, J=6.8 \mathrm{~Hz}, \mathrm{CH}\left(\mathrm{CH}_{3}\right)_{2}\right) .{ }^{13} \mathrm{C} \mathrm{NMR}$ $\left(\mathrm{CDCl}_{3}, 100 \mathrm{MHz}\right): \delta 139.9,129.2,127.9,127.8,81.4,44.7,38.9,33.6,28.0,25.0,23.0,22.7$. $[\alpha]_{\mathrm{D}}{ }^{20}-9.78^{\circ}\left(c=1.53, \mathrm{CHCl}_{3}\right)$ for a sample that is $95 \%$ ee. 
The optical purity of 6-methyl-1-nitro-2-phenylheptane is established by chiral GLC analysis (Chiraldex GTA, $140^{\circ} \mathrm{C}, 15 \mathrm{psi}$ ); chromatograms are illustrated below for a $95 \%$ ee sample:
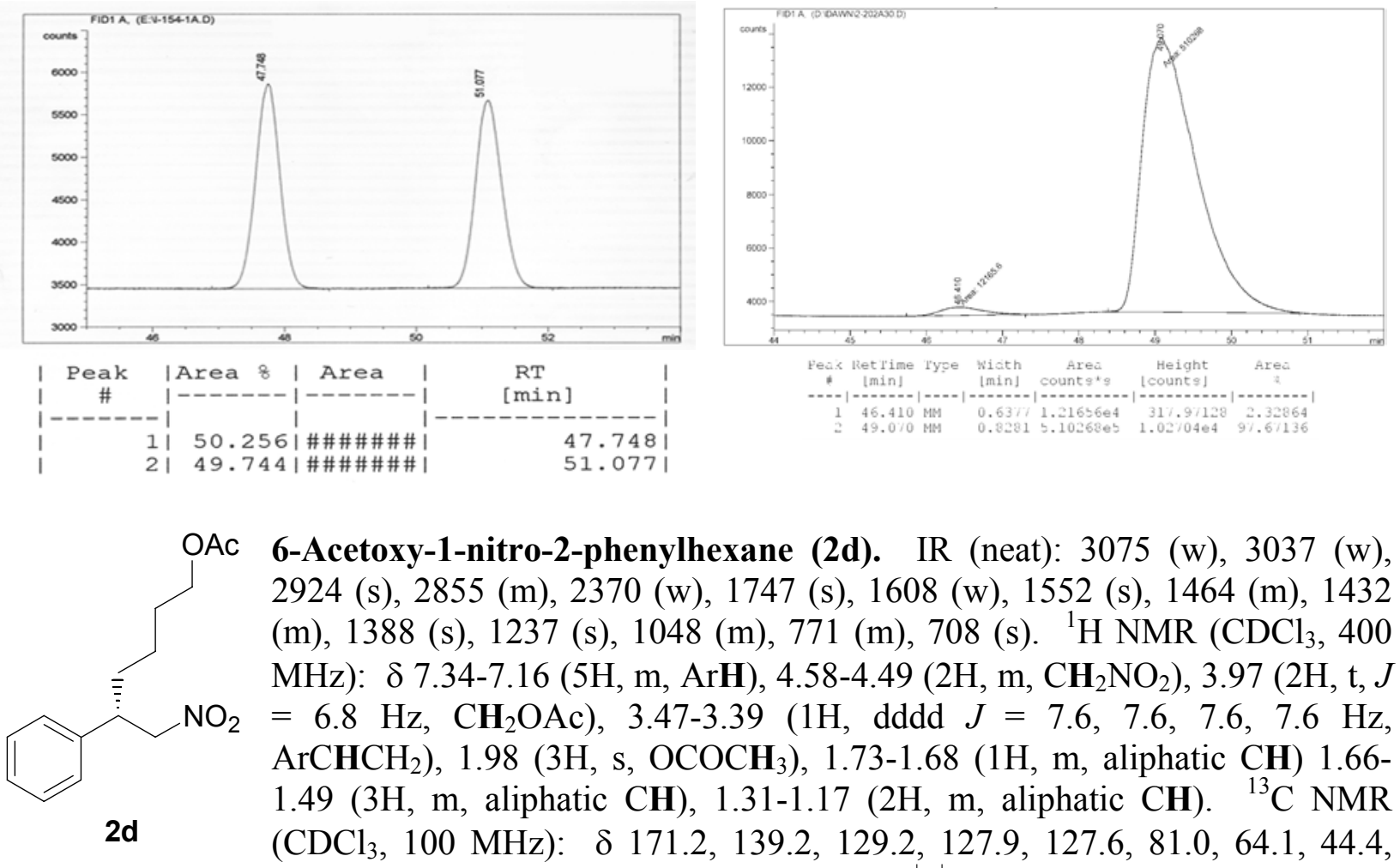
32.7, 28.4, 23.5, 21.1. HRMS Calcd. for $\mathrm{C}_{14} \mathrm{H}_{19} \mathrm{NO}_{4} \mathrm{Na}\left[\mathrm{M}+\mathrm{Na}^{+}\right]^{+}$: 288.1212. Found: 288.1210 . Anal. Calcd. for $\mathrm{C}_{14} \mathrm{H}_{19} \mathrm{NO}_{4}$ : C, 63.38; H, 7.22; N, 5.28. Found: C, 63.16; H, 7.47; N, 4.99. $[\alpha]_{\mathrm{D}}{ }^{20}-12.2^{\circ}\left(c=0.207, \mathrm{CHCl}_{3}\right)$ for a sample that is $89 \%$ ee.

The optical purity of 1-acetoxy-6-nitro-5-phenylhexane is established by chiral HPLC analysis (Chiral Technologies Chiralpak OD, $254 \mathrm{~nm}, 97: 3$ hexanes/i-PrOH, $1 \mathrm{~mL} / \mathrm{min}$ ); chromatograms are illustrated below for a $89 \%$ ee sample:
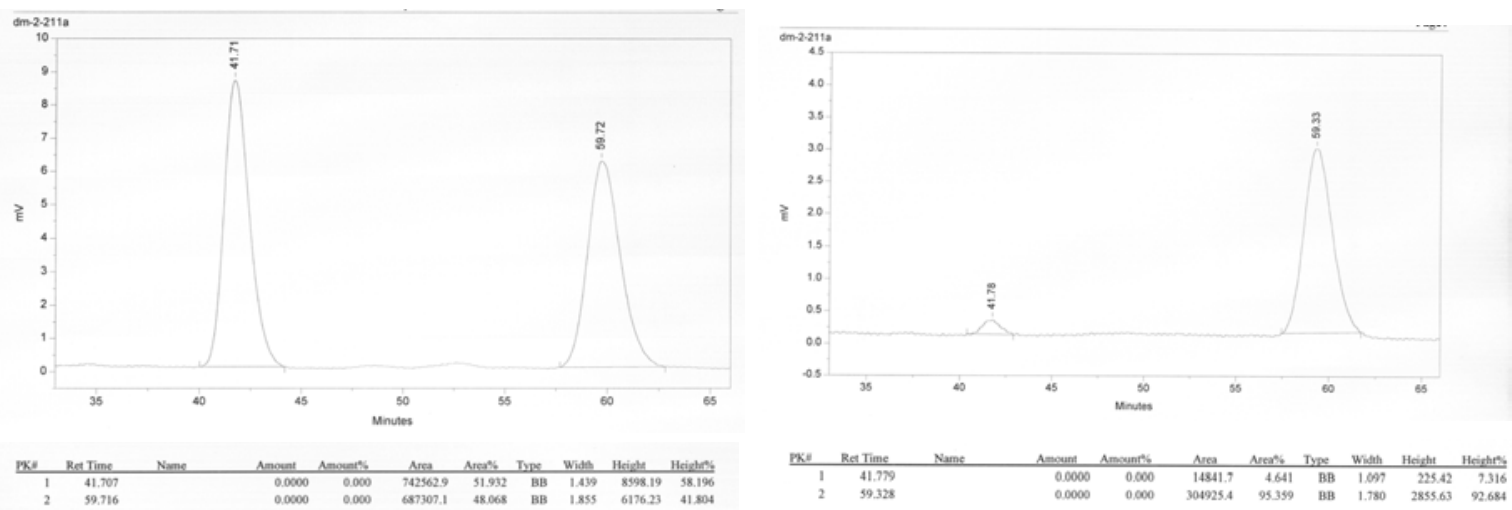


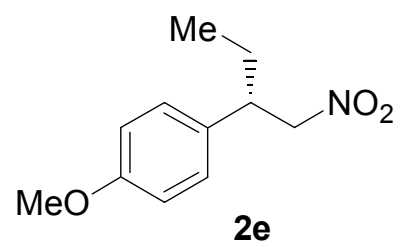

1-Nitro-2-(4-methoxyphenyl)butane (2e). IR (neat): 2962 (s), 2936 (s), $2880(\mathrm{~m}), 2842(\mathrm{~m}), 2754(\mathrm{w}), 2552(\mathrm{w}), 2068(\mathrm{~m}), 1898(\mathrm{~m}), 1747$ (w), 1621 (s), 1558 (s), 1514 (s), 1464 (s), 1388 (s), 1313 (s), 1262 (s), 1040 (s), 834 (s) $690(\mathrm{~m}) .{ }^{1} \mathrm{H}$ NMR $\left(\mathrm{CDCl}_{3}, 400 \mathrm{MHz}\right): \delta$ 7.10-7.07 $(2 \mathrm{H}, \mathrm{m}, \mathrm{ArH}), 6.87-6.83(2 \mathrm{H}, \mathrm{m}, \mathrm{ArH}), 4.56-4.45\left(2 \mathrm{H}, \mathrm{m}, \mathrm{CH}_{2} \mathrm{NO}_{2}\right)$, $3.77\left(3 \mathrm{H}, \mathrm{s}, \mathrm{OCH}_{3}\right), 3.33-3.25\left(1 \mathrm{H}, \mathrm{m}, \mathrm{ArCHCH}{ }_{2}\right), 1.77-1.56(1 \mathrm{H}, \mathrm{dqd}$, $\left.J=14.8,7.6,5.2 \mathrm{~Hz}, \mathrm{CHHCH}_{3}\right), 1.77-1.56\left(1 \mathrm{H}, \mathrm{dqd}, J=14.4,9.6,7.6 \mathrm{~Hz}, \mathrm{CHHCH}_{3}\right), 0.82(3 \mathrm{H}$, $\left.\mathrm{t}, J=7.2 \mathrm{~Hz}, \mathrm{CH}_{2} \mathrm{CH}_{3}\right) .{ }^{13} \mathrm{C} \mathrm{NMR}\left(\mathrm{CDCl}_{3}, 100 \mathrm{MHz}\right): \delta 159.0,131.3,128.7,114.4,81.1,55.3$, 45.4, 26.3, 11.7. HRMS Calcd. for $\mathrm{C}_{11} \mathrm{H}_{15} \mathrm{NO}_{3}$ : 209.1052. Found: 209.1060 . $\left.\alpha\right]_{\mathrm{D}}{ }^{20}-37.8^{\circ}(c=$ $1.51, \mathrm{CHCl}_{3}$ ) for a sample that is $95 \%$ ee.

The optical purity of 1-nitro-2-(4-methoxyphenyl)butane is established by chiral GLC analysis (Chiraldex GTA, $115{ }^{\circ} \mathrm{C}, 15 \mathrm{psi}$ ); chromatograms are illustrated below for a $95 \%$ ee sample:
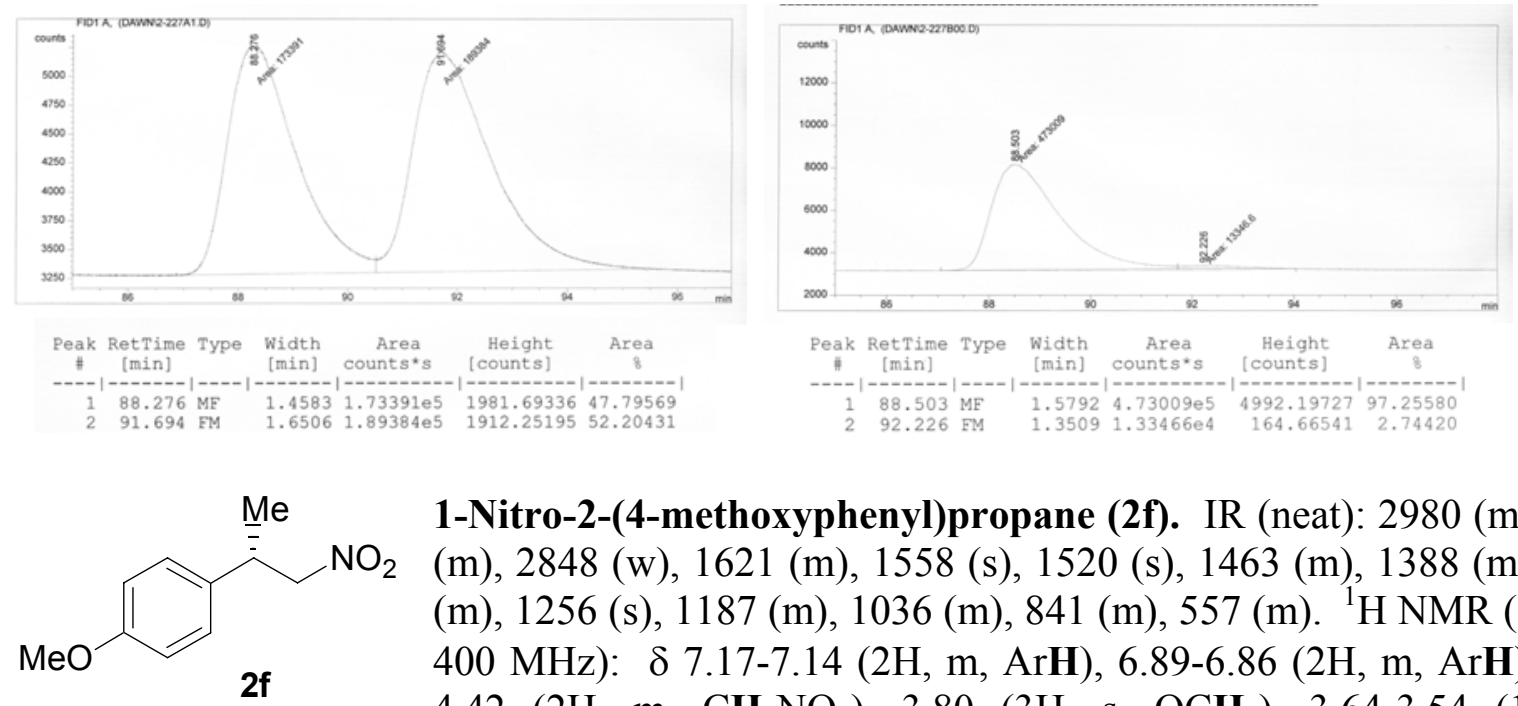

1-Nitro-2-(4-methoxyphenyl)propane (2f). IR (neat): 2980 (m), 2936 (m), $2848(\mathrm{w}), 1621(\mathrm{~m}), 1558(\mathrm{~s}), 1520(\mathrm{~s}), 1463(\mathrm{~m}), 1388(\mathrm{~m}), 1312$ (m), 1256 (s), $1187(\mathrm{~m}), 1036(\mathrm{~m}), 841(\mathrm{~m}), 557(\mathrm{~m}) .{ }^{1} \mathrm{H} \mathrm{NMR}\left(\mathrm{CDCl}_{3}\right.$, $400 \mathrm{MHz}): \delta$ 7.17-7.14 (2H, m, ArH), 6.89-6.86 (2H, m, ArH), 4.54$4.42\left(2 \mathrm{H}, \mathrm{m}, \mathrm{CH}_{2} \mathrm{NO}_{2}\right), 3.80\left(3 \mathrm{H}, \mathrm{s}, \mathrm{OCH}_{3}\right), 3.64-3.54(1 \mathrm{H}, \mathrm{m}$, $\left.\mathrm{ArCHCH}_{2}\right), 1.36\left(3 \mathrm{H}, \mathrm{d}, J=7.2 \mathrm{~Hz}, \mathrm{CHCH}_{3}\right) .{ }^{13} \mathrm{C} \mathrm{NMR}\left(\mathrm{CDCl}_{3}, 100 \mathrm{MHz}\right): \delta 159.0,133.0$, 128.0, 114.5, 82.3, 55.4, 38.1, 18.9. HRMS Calcd. for $\mathrm{C}_{10} \mathrm{H}_{13} \mathrm{NO}_{3}$ : 195.0895. Found: 195.0898. $[\alpha]_{\mathrm{D}}{ }^{20}-48.4^{\circ}\left(c=0.627, \mathrm{CHCl}_{3}\right)$ for a sample that is $95 \%$ ee.

The optical purity of 1-nitro-2-(4-methoxyphenyl)propane is established by chiral HPLC analysis (Chiraldex GTA, $120^{\circ} \mathrm{C}, 15 \mathrm{psi}$ ); chromatograms are illustrated below for a 95\% ee sample: 

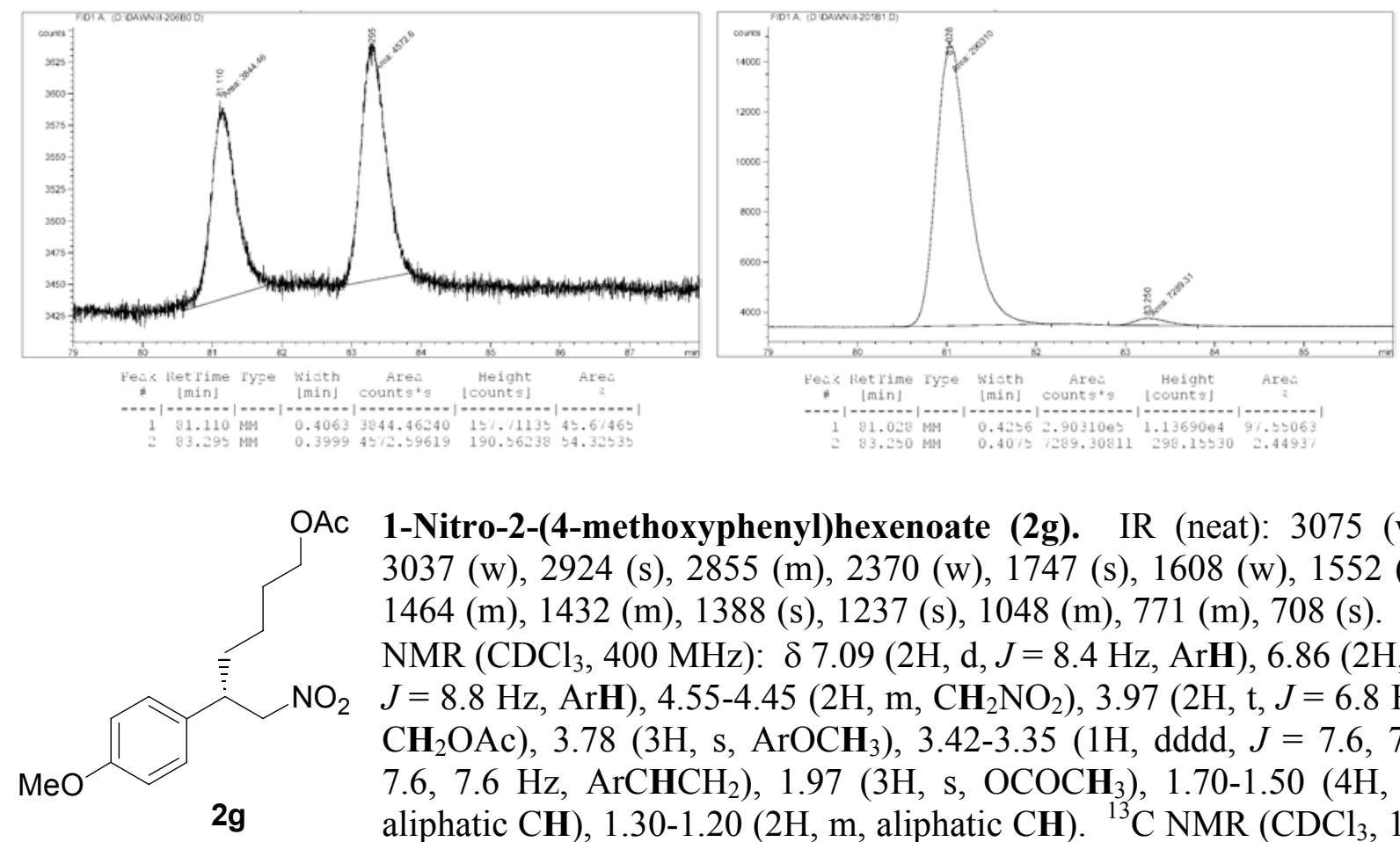

1-Nitro-2-(4-methoxyphenyl)hexenoate (2g). IR (neat): 3075 (w), 3037 (w), 2924 (s), 2855 (m), 2370 (w), 1747 (s), 1608 (w), 1552 (s), 1464 (m), 1432 (m), 1388 (s), 1237 (s), 1048 (m), 771 (m), 708 (s). ${ }^{1} \mathrm{H}$ NMR $\left(\mathrm{CDCl}_{3}, 400 \mathrm{MHz}\right): \delta 7.09(2 \mathrm{H}, \mathrm{d}, J=8.4 \mathrm{~Hz}, \mathrm{ArH}), 6.86(2 \mathrm{H}, \mathrm{d}$, $J=8.8 \mathrm{~Hz}, \mathrm{ArH}), 4.55-4.45\left(2 \mathrm{H}, \mathrm{m}, \mathrm{CH}_{2} \mathrm{NO}_{2}\right), 3.97(2 \mathrm{H}, \mathrm{t}, J=6.8 \mathrm{~Hz}$, $\left.\mathrm{CH}_{2} \mathrm{OAc}\right), 3.78(3 \mathrm{H}, \mathrm{s}, \operatorname{ArOCH})_{3}, 3.42-3.35(1 \mathrm{H}, \mathrm{dddd}, J=7.6,7.6$, 7.6, 7.6 Hz, $\operatorname{ArCHCH} 2), 1.97\left(3 \mathrm{H}, \mathrm{s}, \mathrm{OCOCH}_{3}\right), 1.70-1.50(4 \mathrm{H}, \mathrm{m}$, aliphatic $\mathrm{CH}), 1.30-1.20(2 \mathrm{H}, \mathrm{m}$, aliphatic $\mathrm{CH}) .{ }^{13} \mathrm{C} \mathrm{NMR}\left(\mathrm{CDCl}_{3}, 100\right.$ MHz): $\delta 171.2,159.1,131.0,128.6,114.5,81.2,64.1,55.4,43.6,32.7,28.3,23.4,21.0$. HRMS Calcd. for $\mathrm{C}_{15} \mathrm{H}_{21} \mathrm{NO}_{5}$ : 295.1420. Found: 295.1419. $[\alpha]_{\mathrm{D}}{ }^{20}-12.2^{\circ}\left(c=1.50, \mathrm{CHCl}_{3}\right)$ for a sample that is $93 \%$ ee.

The optical purity of the 1-nitro-2-(4-methoxyphenyl)hexenoate is established by chiral HPLC analysis (Chiral Technologies Chiralpak OD, 254 nm, 97:3 hexanes/ $i$-PrOH, $1 \mathrm{~mL} / \mathrm{min}$ ); chromatograms are illustrated below for a $93 \%$ ee sample:
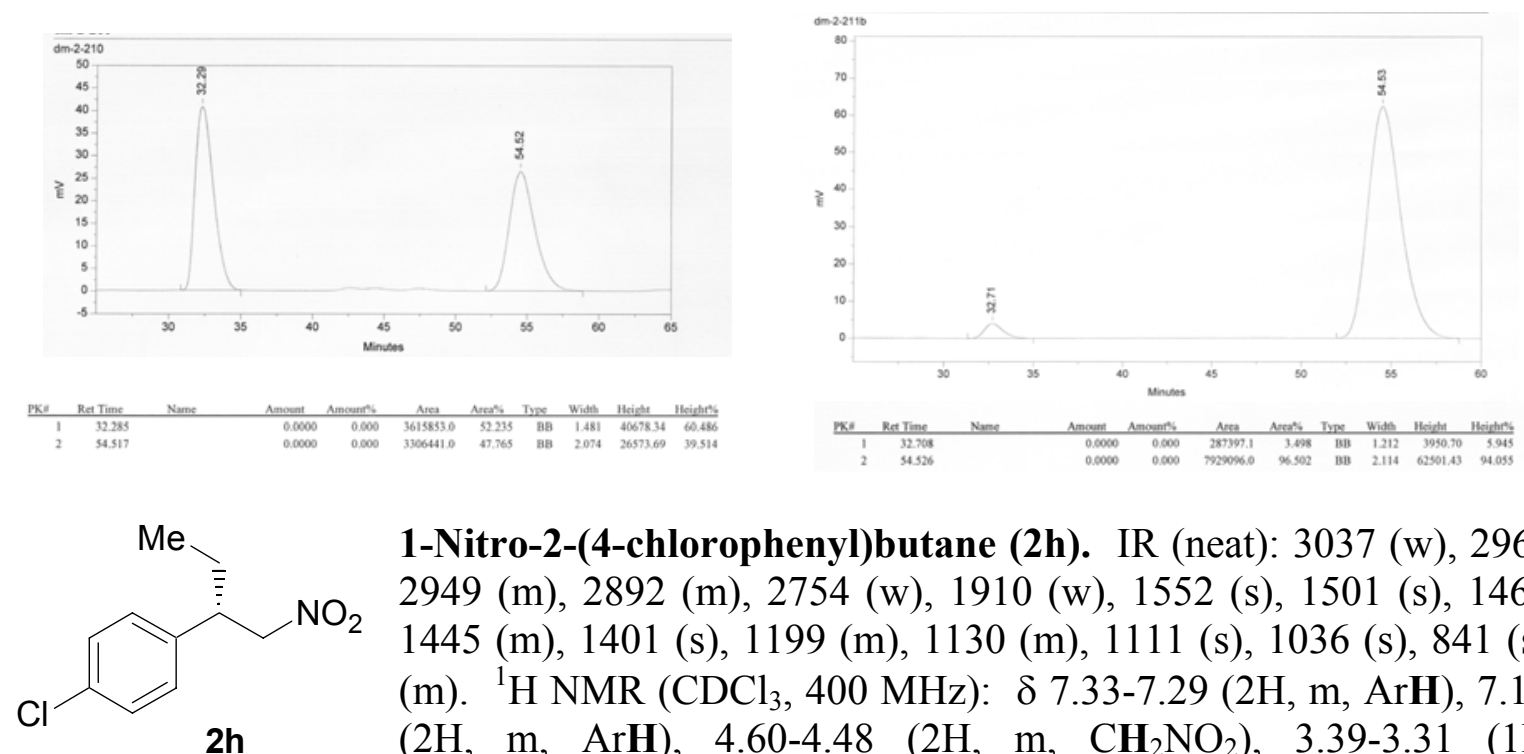

1-Nitro-2-(4-chlorophenyl)butane (2h). IR (neat): 3037 (w), 2968 (m), 2949 (m), $2892(\mathrm{~m}), 2754(\mathrm{w}), 1910(\mathrm{w}), 1552$ (s), 1501 (s), $1464(\mathrm{~m})$, $1445(\mathrm{~m}), 1401(\mathrm{~s}), 1199(\mathrm{~m}), 1130(\mathrm{~m}), 1111(\mathrm{~s}), 1036(\mathrm{~s}), 841$ (s), 740 (m). ${ }^{1} \mathrm{H} \mathrm{NMR}\left(\mathrm{CDCl}_{3}, 400 \mathrm{MHz}\right): \delta$ 7.33-7.29 (2H, m, ArH), 7.14-7.11 $(2 \mathrm{H}, \mathrm{m}, \mathrm{ArH}), 4.60-4.48\left(2 \mathrm{H}, \mathrm{m}, \mathrm{CH}_{2} \mathrm{NO}_{2}\right), 3.39-3.31(1 \mathrm{H}, \mathrm{m}$, $\left.\operatorname{ArCHCH}_{2}\right), 1.81-1.60\left(1 \mathrm{H}, \mathrm{dqd}, J=14.8,7.6,5.6 \mathrm{~Hz}, \mathrm{CHHCH}_{3}\right), 1.81-1.60(1 \mathrm{H}, \mathrm{dqd}, J=14.4$, 9.6, 7.2 Hz, $\left.\mathrm{CHHCH}_{3}\right), 0.84\left(3 \mathrm{H}, \mathrm{t}, J=7.2 \mathrm{~Hz}, \mathrm{CH}_{2} \mathrm{CH}_{3}\right) .{ }^{13} \mathrm{C} \mathrm{NMR}\left(\mathrm{CDCl}_{3}, 100 \mathrm{MHz}\right): \delta$ 137.9, 133.5, 129.2, 129.1, 80.6, 45.6, 26.3, 11.6. HRMS Calcd. for $\mathrm{C}_{10} \mathrm{H}_{12} \mathrm{ClNO}_{2}$ : 213.0557. 
Found: 213.0553. Anal. Calcd. for $\mathrm{C}_{10} \mathrm{H}_{12} \mathrm{NO}_{2}$ : C, 56.21; H, 5.66; N, 6.56. Found: C, 56.42; H, $5.58 ; \mathrm{N}, 6.59 .[\alpha]_{\mathrm{D}}{ }^{20}-22.9^{\circ}\left(c=1.20, \mathrm{CHCl}_{3}\right)$ for a sample that is $93 \%$ ee.

The optical purity of 1-nitro-2-(4-chlorophenyl)butane is established by chiral GLC analysis (Chiraldex GTA, $120^{\circ} \mathrm{C}, 15 \mathrm{psi}$ ); chromatograms are illustrated below for a $93 \%$ ee sample:
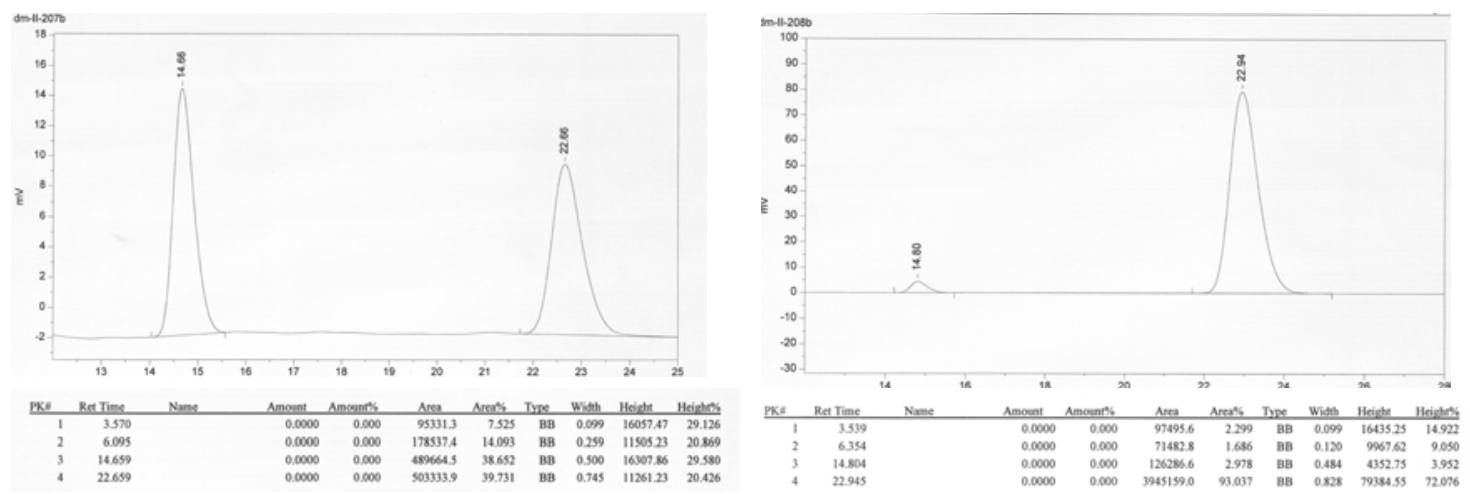

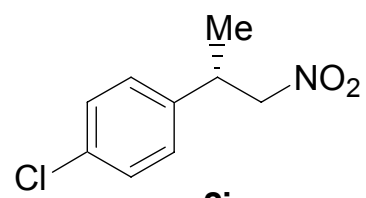

2i

1-Nitro-2-(4-chlorophenyl)propane (2i). IR (neat): 3037 (w), 2980 (m), $2943(\mathrm{~m}), 2880(\mathrm{w}), 1917(\mathrm{w}), 1734(\mathrm{~m}), 1558(\mathrm{~s}), 1501(\mathrm{~s}), 1464(\mathrm{~m})$, $1432(\mathrm{~m}), 1413(\mathrm{~m}), 1382(\mathrm{~s}), 1350(\mathrm{w}), 1281(\mathrm{w}), 1199(\mathrm{w}), 1099(\mathrm{~s})$, 1023 (s), $910(\mathrm{w}), 847$ (s), $790(\mathrm{w}), 752(\mathrm{~m}), 645(\mathrm{~m}) .{ }^{1} \mathrm{H} \mathrm{NMR}\left(\mathrm{CDCl}_{3}\right.$, $400 \mathrm{MHz}): \delta$ 7.33-7.30 (2H, m, ArH), 7.18-7.15 (2H, m, ArH), 4.54-4.45 $\left(2 \mathrm{H}, \mathrm{m}, \mathrm{CH}_{2} \mathrm{NO}_{2}\right), 3.67-3.58(1 \mathrm{H}, \mathrm{m}, \mathrm{ArCHCH}), 1.36\left(3 \mathrm{H}, \mathrm{d}, J=7.2 \mathrm{~Hz}, \mathrm{CHCH}_{3}\right) .{ }^{13} \mathrm{C} \mathrm{NMR}$ $\left(\mathrm{CDCl}_{3}, 100 \mathrm{MHz}\right): \delta 139.5,133.5,129.3,128.4,81.7,38.2,18.8$. HRMS Calcd. for $\mathrm{C}_{9} \mathrm{H}_{10} \mathrm{ClNO}_{2}$ : 199.0400. Found: 199.0398. Anal. Calcd. for $\mathrm{C}_{9} \mathrm{H}_{10} \mathrm{NO}_{2}: \mathrm{C}, 54.15 ; \mathrm{H}, 5.05 ; \mathrm{N}$, 7.02. Found: $\mathrm{C}, 54.41 ; \mathrm{H}, 5.12 ; \mathrm{N}, 7.33 .[\alpha]_{\mathrm{D}}{ }^{20}-40.0^{\circ}\left(c=1.45, \mathrm{CHCl}_{3}\right)$ for a sample that is $93 \%$ ee.

The optical purity of the 1-nitro-2-(4-chlorophenyl)propane is established by chiral GLC analysis (Chiraldex GTA, $130^{\circ} \mathrm{C}, 15 \mathrm{psi}$ ); chromatograms are illustrated below for a $94 \%$ ee sample:
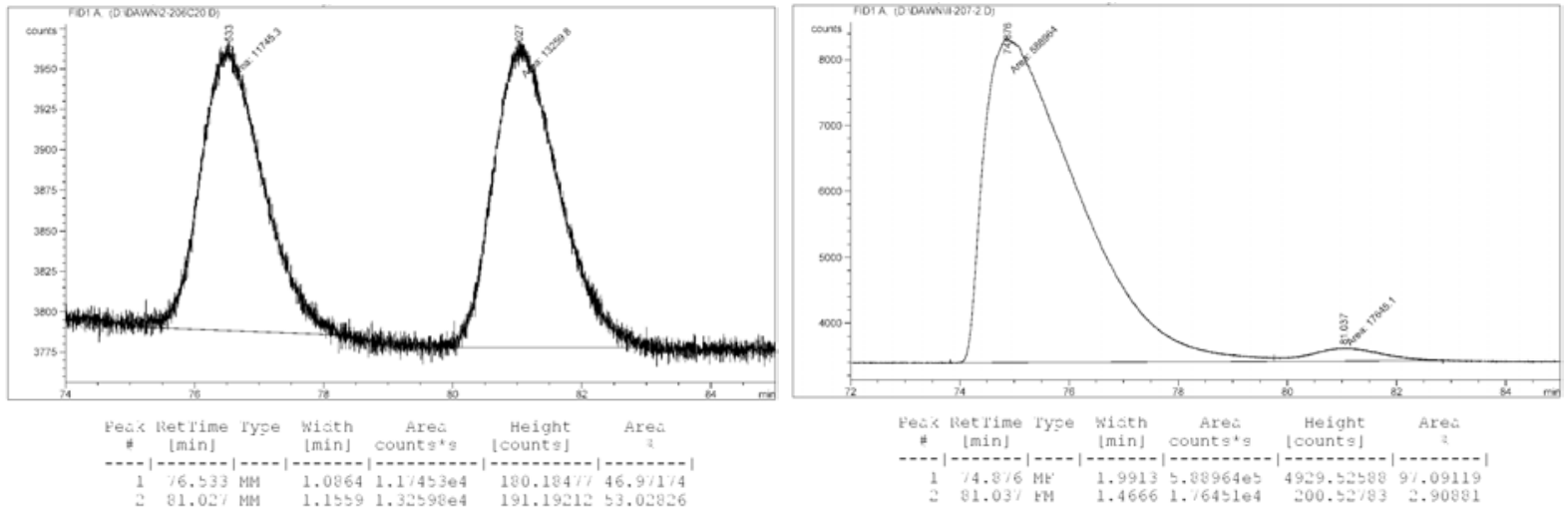


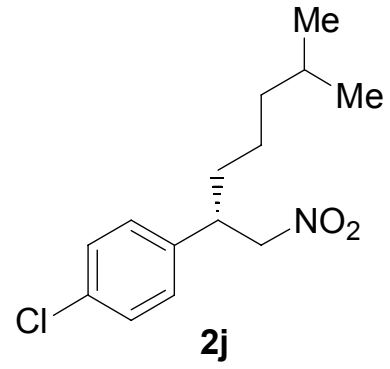

27.8, 24.7, 22.8, 22.5. HRMS Calcd. for $\mathrm{C}_{14} \mathrm{H}_{20} \mathrm{ClNO}_{2}$ : 269.1183. Found: 269.1185. $[\alpha]_{\mathrm{D}}{ }^{20}$ $-3.05^{\circ}\left(c=0.940, \mathrm{CHCl}_{3}\right)$ for a sample that is of $90 \%$ ee.

The optical purity of 1-nitro-2-(4-chlorophenyl-5-methyl)heptane is established by chiral GLC analysis (Chiraldex GTA, $120^{\circ} \mathrm{C}, 15 \mathrm{psi}$ ); chromatograms are illustrated below for an $90 \%$ ee sample:
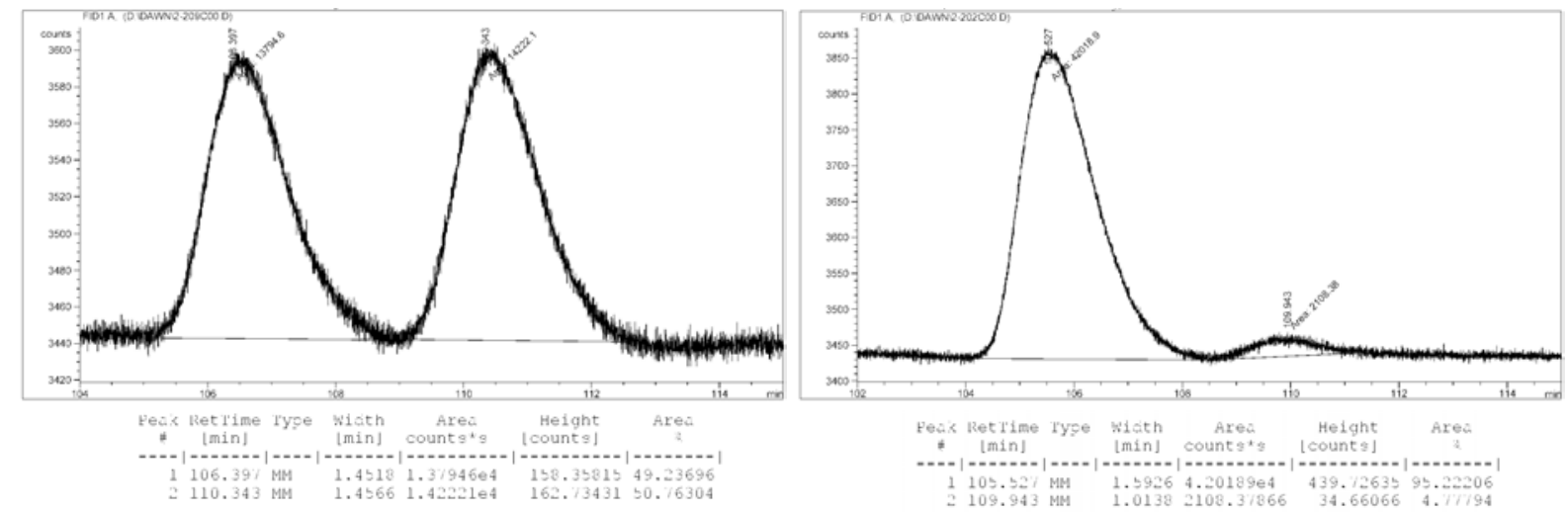

6-methyl-1-nitro-2-(4-chlorophenyl)heptane (2j). IR (neat): 2955 (m), $2930(\mathrm{~m}), 2873(\mathrm{~m}), 2864(\mathrm{w}), 1558(\mathrm{~s}), 1489(\mathrm{~m}), 1464(\mathrm{w}), 1432$ (w), $1388(\mathrm{~m}), 1098(\mathrm{~m}), 1023(\mathrm{~m}), 834(\mathrm{~m}) .{ }^{1} \mathrm{H}$ NMR $\left(\mathrm{CDCl}_{3}, 400\right.$ $\mathrm{MHz}): \quad \delta$ 7.32-7.28 (2H, m, ArH), 7.14-7.10 (2H, m, ArH), 4.58-4.46 $\left(2 \mathrm{H}, \mathrm{m}, \mathrm{CH}_{2} \mathrm{NO}_{2}\right), 3.39-3.31(1 \mathrm{H}, \mathrm{m}, \operatorname{ArCHCH})_{2}, 1.66-1.59(2 \mathrm{H}, \mathrm{m}$, CHCH $\left.{ }_{2}\left(\mathrm{CH}_{2}\right)_{2} \mathrm{CH}\left(\mathrm{CH}_{3}\right)_{2}\right), 1.50-1.41\left(1 \mathrm{H}, \mathrm{m}, \mathrm{CH}\left(\mathrm{CH}_{3}\right)_{2}\right), 1.21-1.10(4 \mathrm{H}$, $\mathrm{m}$, aliphatic $\mathrm{CH}), 0.81\left(6 \mathrm{H}, \mathrm{d}, J=7.2 \mathrm{~Hz}, \mathrm{CH}\left(\mathrm{CH}_{3}\right)_{2}\right) .{ }^{13} \mathrm{C} \mathrm{NMR}$ $\left(\mathrm{CDCl}_{3}, 100 \mathrm{MHz}\right): \delta 138.2,133.5,129.3,129.0,80.1,43.9,38.6,33.3$,

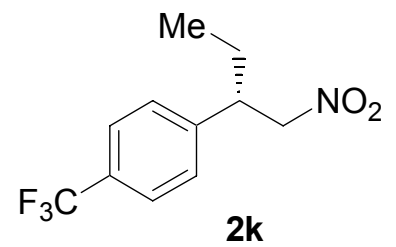

1-Nitro-2-(4-trifluoromethylphenyl)butane (2k). IR (neat): 2980 (m), $2949(\mathrm{~m}), 2880(\mathrm{~m}), 1923(\mathrm{w}), 1633(\mathrm{~m}), 1558(\mathrm{~s}), 1464(\mathrm{w}), 1426(\mathrm{~m})$, $1380(\mathrm{~s}), 1331$ (s), $1174(\mathrm{~s}), 1130(\mathrm{~s}), 1073$ (s), $1029(\mathrm{~m}), 853(\mathrm{~m}), 721$ (w), $620(\mathrm{w}) .{ }^{1} \mathrm{H}$ NMR $\left(\mathrm{CDCl}_{3}, 400 \mathrm{MHz}\right): \delta 7.59(2 \mathrm{H}, \mathrm{d}, J=8.4 \mathrm{~Hz}$, ArH), $7.30(2 \mathrm{H}, \mathrm{d}, J=8.4 \mathrm{~Hz}, \mathrm{ArH}), 4.63-4.52\left(2 \mathrm{H}, \mathrm{m}, \mathrm{CH}_{2} \mathrm{NO}_{2}\right), 3.47-$ $3.40(1 \mathrm{H}, \mathrm{m}, \mathrm{ArCHCH} 2), 1.84-1.63\left(2 \mathrm{H}, \mathrm{dqd}, J=14.8,7.6,5.2 \mathrm{~Hz}, \mathrm{CHHCH}_{3}\right), 1.80-1.60(2 \mathrm{H}$, dqd, $\left.J=14.4,9.6,7.2 \mathrm{~Hz}, \mathrm{CH}_{2} \mathrm{CH}_{3}\right), 0.84\left(3 \mathrm{H}, \mathrm{t}, J=7.6 \mathrm{~Hz}, \mathrm{CH}_{2} \mathrm{CH}_{3}\right) .{ }^{13} \mathrm{C}$ NMR $\left(\mathrm{CDCl}_{3}, 100\right.$ $\mathrm{MHz}): \quad \delta 143.7,130.2\left(1 \mathrm{C}\right.$, dddd, $\left.J=70,70,40,40 \mathrm{~Hz}, \mathrm{ArCF}_{3}\right), 128.2,126.1,125.5,122.8$, 80.4, 46.0, 26.4, 11.7. HRMS Calcd. for $\mathrm{C}_{11} \mathrm{H}_{12} \mathrm{~F}_{3} \mathrm{NO}_{2}$ : 247.0820. Found: 247.0825. [ $\left.\alpha\right]_{\mathrm{D}}{ }^{20}$ $-18.4^{\circ}\left(c=1.15, \mathrm{CHCl}_{3}\right)$ for a sample that is $79 \%$ ee.

The optical purity of 1-nitro-2-(4-trifluoromethylphenyl)butane is established by chiral HPLC analysis (Chiral Technologies Chiralpak OD, $254 \mathrm{~nm}, 97: 3$ hexanes/ $i-\mathrm{PrOH}, 1 \mathrm{~mL} / \mathrm{min}$ ); chromatograms are illustrated below for a $79 \%$ ee sample: 

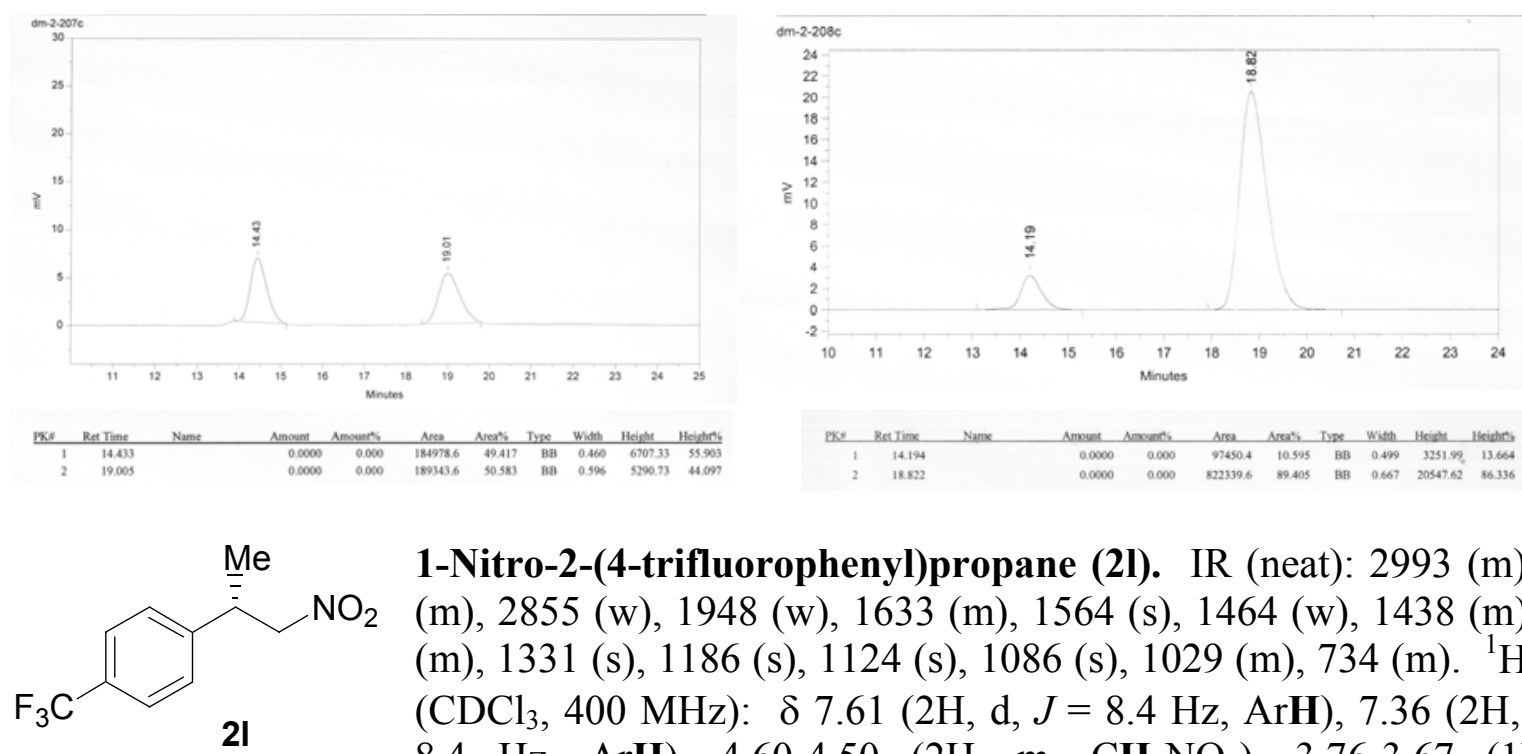

1-Nitro-2-(4-trifluorophenyl)propane (2l). IR (neat): 2993 (m), 2930 (m), $2855(\mathrm{w}), 1948(\mathrm{w}), 1633(\mathrm{~m}), 1564(\mathrm{~s}), 1464(\mathrm{w}), 1438(\mathrm{~m}), 1394$ (m), 1331 (s), 1186 (s), 1124 (s), 1086 (s), 1029 (m), 734 (m). ${ }^{1} \mathrm{H}$ NMR $\left(\mathrm{CDCl}_{3}, 400 \mathrm{MHz}\right): \delta 7.61(2 \mathrm{H}, \mathrm{d}, J=8.4 \mathrm{~Hz}, \mathrm{ArH}), 7.36(2 \mathrm{H}, \mathrm{d}, J=$ $8.4 \mathrm{~Hz}, \mathrm{ArH}), 4.60-4.50\left(2 \mathrm{H}, \mathrm{m}, \mathrm{CH}_{2} \mathrm{NO}_{2}\right), 3.76-3.67(1 \mathrm{H}, \mathrm{m}$, $\left.\mathrm{ArCHCH}_{2}\right), 1.40\left(3 \mathrm{H}, \mathrm{d}, J=7.2 \mathrm{~Hz}, \mathrm{CHCH}_{3}\right) .{ }^{13} \mathrm{C} \mathrm{NMR}\left(\mathrm{CDCl}_{3}, 100 \mathrm{MHz}\right): \delta 145.1,130.2$ (1C, dddd, $\left.J=70,70,40,40 \mathrm{~Hz}, \mathrm{ArCF}_{3}\right), 127.5,126.1,125.5,122.8,81.4,38.6,18.8$. HRMS Calcd. for $\mathrm{C}_{10} \mathrm{H}_{10} \mathrm{~F}_{3} \mathrm{NO}_{2}$ : 233.0664. Found: 233.0660. Anal. Calcd. for $\mathrm{C}_{10} \mathrm{H}_{10} \mathrm{~F}_{3} \mathrm{NO}_{2}: \mathrm{C}, 51.51$; $\mathrm{H}, 4.32 ; \mathrm{N}, 6.01$. Found: $\mathrm{C}, 51.34 ; \mathrm{H}, 4.33 ; \mathrm{N}, 5.91 .[\alpha]_{\mathrm{D}}{ }^{20}-24.8^{\circ}\left(c=0.213, \mathrm{CHCl}_{3}\right)$ for a sample that is $95 \%$ ee.

The optical purity of 1-nitro-2-(4-trifluorophenyl)propane is established by chiral GLC analysis ( $\alpha$-dex, $110^{\circ} \mathrm{C}, 15 \mathrm{psi}$ ); chromatograms are illustrated below for a $95 \%$ ee sample:
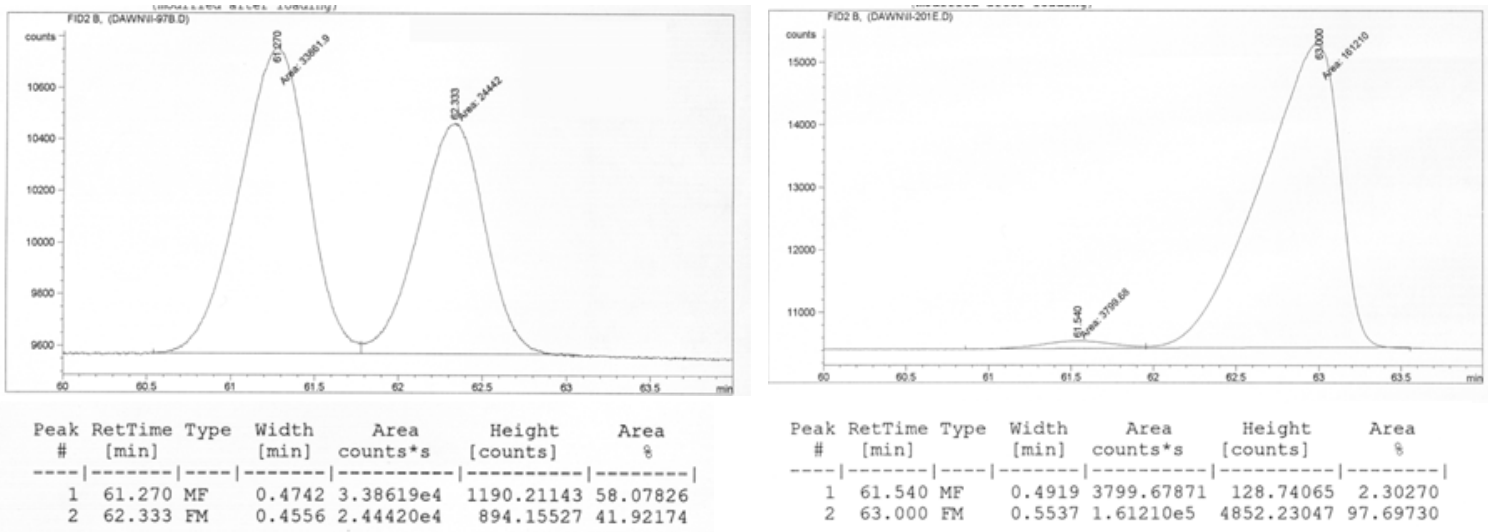

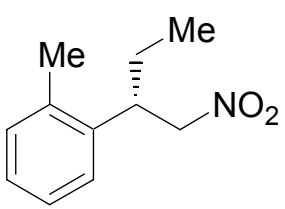

$2 \mathrm{~m}$

1-Nitro-2-(2-methylphenyl)butane (2m). IR (neat): 3062 (w), 3037 (w), 2974 (m), 2943 (m), 2880 (w), 1558 (s), 1507 (m), 1476 (m), 1432 (m), 1382 (m), $1136(\mathrm{~m}), 777(\mathrm{~m}), 734(\mathrm{~m}) .{ }^{1} \mathrm{H} \mathrm{NMR}\left(\mathrm{CDCl}_{3}, 400 \mathrm{MHz}\right): \delta$ 7.26-7.13 $(4 \mathrm{H}, \mathrm{m}, \mathrm{ArH}), 4.60-4.50\left(2 \mathrm{H}, \mathrm{m}, \mathrm{CH}_{2} \mathrm{NO}_{2}\right), 3.79-3.70\left(1 \mathrm{H}, \mathrm{m}, \mathrm{ArCHCH}_{2}\right)$, $2.39\left(3 \mathrm{H}, \mathrm{s}, \mathrm{ArCH}_{3}\right), 1.83-1.64\left(2 \mathrm{H}, \mathrm{m}, \mathrm{CH}_{2} \mathrm{CH}_{3}\right), 0.87-0.82(3 \mathrm{H}, \mathrm{m}$, $\left.\mathrm{CH}_{2} \mathrm{CH}_{3}\right) .{ }^{13} \mathrm{C} \mathrm{NMR}\left(\mathrm{CDCl}_{3}, 100 \mathrm{MHz}\right): \delta 137.7,136.9,131.0,127.2,126.7,125.5,80.4,40.6$, 26.5, 19.8, 11.4. HRMS Calcd. for $\mathrm{C}_{11} \mathrm{H}_{15} \mathrm{NO}_{2}$ : 193.1103. Found: 193.1107. Anal. Calcd. for $\mathrm{C}_{11} \mathrm{H}_{15} \mathrm{NO}_{2}$ : C, 68.37; H, 7.82; N, 7.25. Found: C, 68.42; H, 8.00; N, 7.01. $[\alpha]_{\mathrm{D}}{ }^{20}-15.4^{\circ}(c=$ $1.22, \mathrm{CHCl}_{3}$ ) for a sample that is $94 \%$ ee. 
The optical purity of 1-nitro-2-(2-methylphenyl)butane is established by chiral GLC analysis ( $\beta$-dex $110{ }^{\circ} \mathrm{C}, 15 \mathrm{psi}$ ); chromatograms are illustrated below for a $94 \%$ ee sample:

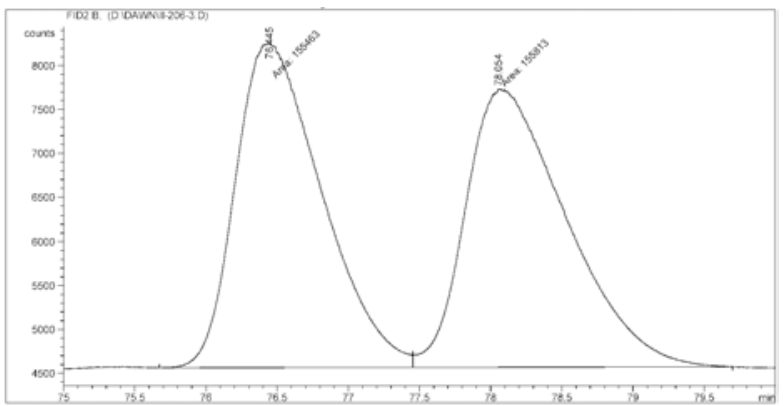

Reus Retrime rype wiath Ares Height Area
[min] countg.g (counts]

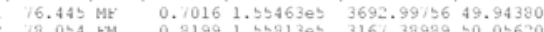

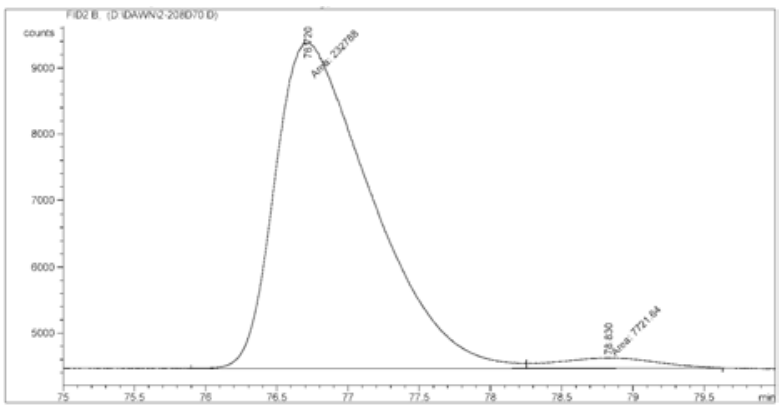

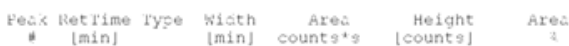

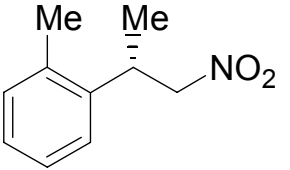

$2 n$

1-Nitro-2-(2-methylphenyl)propane (2n). IR (neat): 3106 (w), 3069 (w), 3024 (m), 2974 (m), 2930 (m), 2880 (w), 1640 (m), 1602 (m), 1564 (s), 1514 (m), $1501(\mathrm{~m}), 1457(\mathrm{~m}), 1438(\mathrm{~m}), 1382(\mathrm{~s}), 1344(\mathrm{~s}), 1300(\mathrm{~m}), 1224(\mathrm{~m})$, 1136 (m), 1099 (w), 1035 (m), 960 (m), 904 (w), 847 (w), 771 (s), 734 (s). ${ }^{1} \mathrm{H}$ NMR $\left(\mathrm{CDCl}_{3}, 400 \mathrm{MHz}\right): \delta$ 7.24-7.15 (4H, m, ArH), 4.58-4.44 (2H, m, $\left.\mathrm{CH}_{2} \mathrm{NO}_{2}\right), 3.97-3.88(1 \mathrm{H}, \mathrm{m}, \operatorname{ArCHCH}), 2.41\left(3 \mathrm{H}, \mathrm{s}, \operatorname{ArCH}_{3}\right), 1.36(3 \mathrm{H}, \mathrm{d}, J=7.2 \mathrm{~Hz}, \mathrm{CHCH})$. ${ }^{13} \mathrm{C} \mathrm{NMR}\left(\mathrm{CDCl}_{3}, 100 \mathrm{MHz}\right): \delta 138.1,137.2,131.3,127.5,127.0,125.4,81.4,34.2,19.6,18.8$. HRMS Calcd. for $\mathrm{C}_{10} \mathrm{H}_{13} \mathrm{NO}_{2}$ : 179.0946 . Found: 179.0946. $[\alpha]_{\mathrm{D}}{ }^{20}-17.8^{\circ} \quad\left(c=0.993, \mathrm{CHCl}_{3}\right)$ for a sample that is $88 \%$ ee.

The optical purity of 1-nitro-2-(2-methylphenyl)propane is established by chiral GLC analysis ( $\beta$-dex $110{ }^{\circ} \mathrm{C}, 15 \mathrm{psi}$ ); chromatograms are illustrated below for an $88 \%$ ee sample:
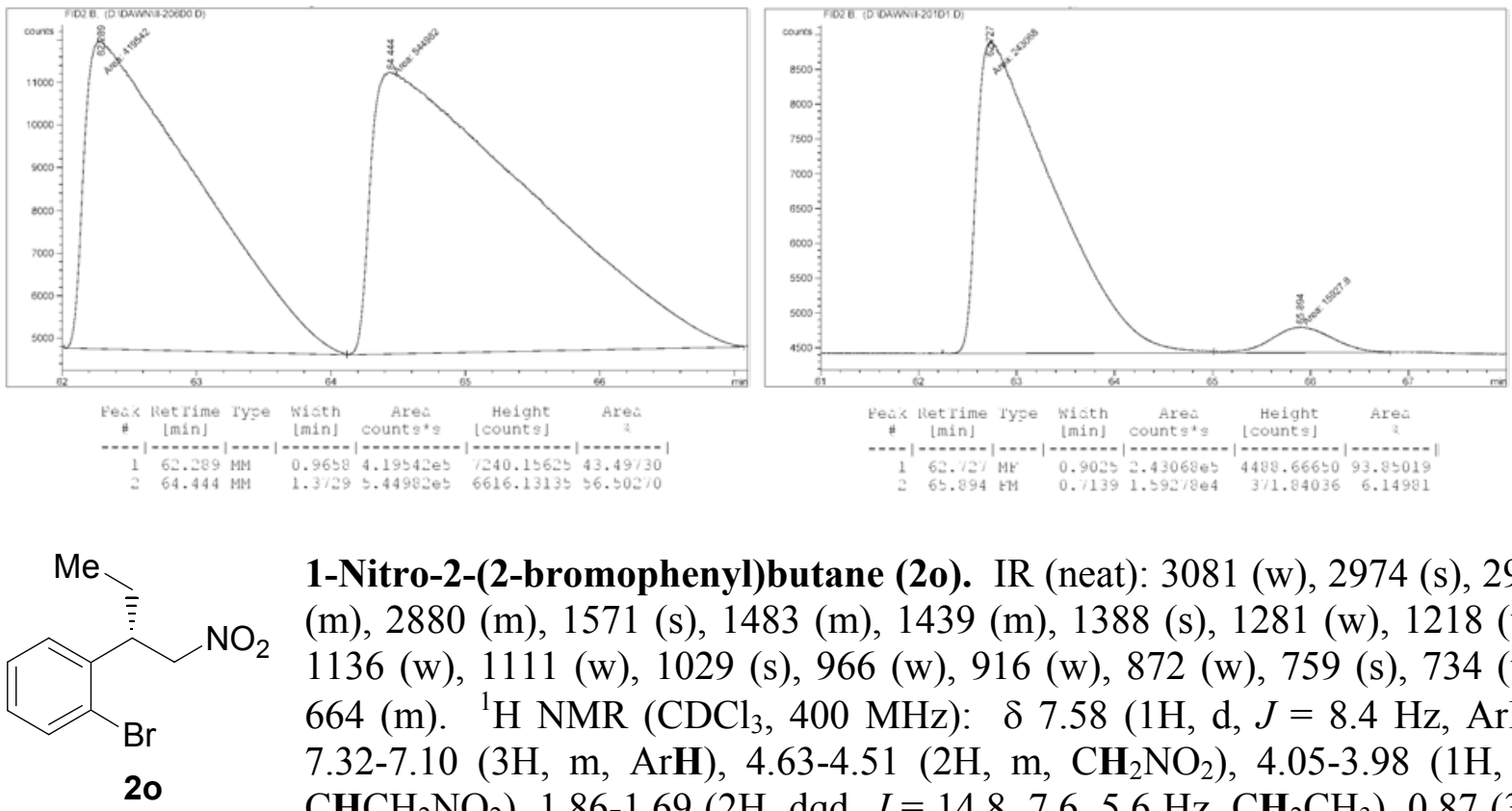

1-Nitro-2-(2-bromophenyl)butane (2o). IR (neat): 3081 (w), 2974 (s), 2936 (m), 2880 (m), 1571 (s), 1483 (m), 1439 (m), $1388(\mathrm{~s}), 1281(\mathrm{w}), 1218(\mathrm{w})$, $1136(\mathrm{w}), 1111(\mathrm{w}), 1029(\mathrm{~s}), 966(\mathrm{w}), 916(\mathrm{w}), 872(\mathrm{w}), 759(\mathrm{~s}), 734(\mathrm{w})$, $664(\mathrm{~m}) .{ }^{1} \mathrm{H}$ NMR $\left(\mathrm{CDCl}_{3}, 400 \mathrm{MHz}\right): \delta 7.58(1 \mathrm{H}, \mathrm{d}, J=8.4 \mathrm{~Hz}, \mathrm{ArH})$, 7.32-7.10 (3H, m, ArH), 4.63-4.51 (2H, m, $\left.\mathrm{CH}_{2} \mathrm{NO}_{2}\right), 4.05-3.98(1 \mathrm{H}, \mathrm{m}$, $\left.\mathrm{CHCH}_{2} \mathrm{NO}_{2}\right), 1.86-1.69$ (2H, dqd, $\left.J=14.8,7.6,5.6 \mathrm{~Hz}, \mathrm{CH}_{2} \mathrm{CH}_{3}\right), 0.87(3 \mathrm{H}$, $\left.\mathrm{t}, J=7.6 \mathrm{~Hz}, \mathrm{CH}_{2} \mathrm{CH}_{3}\right) .{ }^{13} \mathrm{C} \mathrm{NMR}\left(\mathrm{CDCl}_{3}, 100 \mathrm{MHz}\right): \delta 138.4,133.7,129.0,128.1,127.8$, 
125.5, 79.4, 44.1, 25.6, 11.3. HRMS Calcd. for $\mathrm{C}_{10} \mathrm{H}_{12} \mathrm{BrNO}_{2}$ : 257.0051. Found: 257.0054. $[\alpha]_{\mathrm{D}}{ }^{20}-4.07^{\circ}\left(c=1.80, \mathrm{CHCl}_{3}\right)$ for a sample that is $81 \%$ ee.

The optical purity of 1-nitro-2-(2-bromophenyl)butane is established by chiral GLC analysis (Chiraldex GTA, $140{ }^{\circ} \mathrm{C}, 15 \mathrm{psi}$ ) chromatograms are illustrated below for an $81 \%$ ee sample:
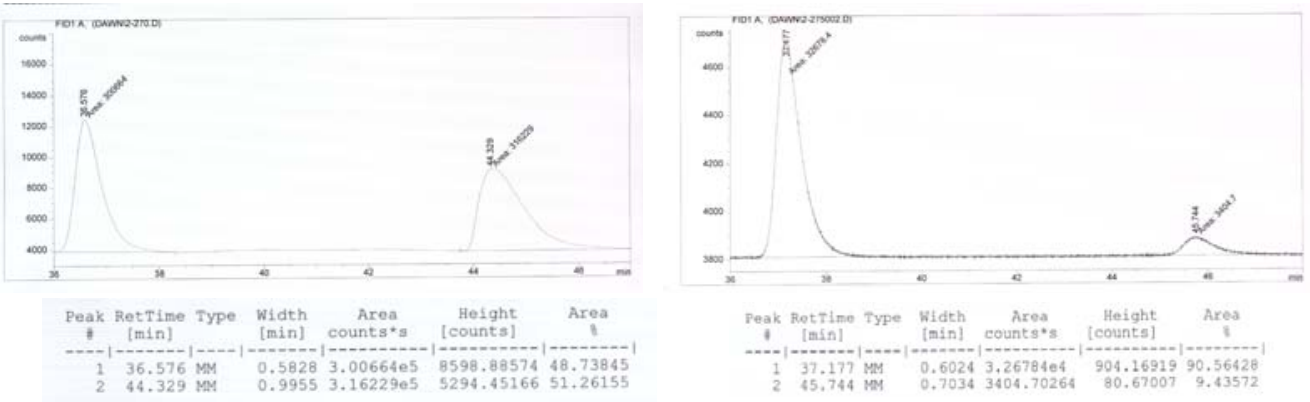

$\begin{array}{ll}\text { 1-Nitro-2-(2-bromophenyl)propane (2p). IR (neat): } 3075(\mathrm{w}), 2993(\mathrm{~m}), \\ 2943(\mathrm{w}), 2886(\mathrm{w}), 1558(\mathrm{~s}), 1482(\mathrm{~m}), 1432(\mathrm{~m}), 1388(\mathrm{~s}), 1281(\mathrm{w}), 1224 \\ \mathrm{Br} & \begin{array}{l}\mathrm{(w}), 1199(\mathrm{w}), 1168(\mathrm{w}), 1111(\mathrm{w}), 1073(\mathrm{w}), 1035(\mathrm{~s}), 948(\mathrm{w}), 910(\mathrm{w}), 759 \\ (\mathrm{~s}), 734(\mathrm{~m}), 664(\mathrm{~m}), 576(\mathrm{w}), 545(\mathrm{w}), 463(\mathrm{~m}),{ }^{1} \mathrm{H} \text { NMR }\left(\mathrm{CDCl}_{3}, 400\right.\end{array}\end{array}$ 2p $\quad \mathrm{MHz}): \delta 7.58(1 \mathrm{H}, \mathrm{d}, J=7.6 \mathrm{~Hz}, \operatorname{ArH}), 7.33-7.11(3 \mathrm{H}, \mathrm{m}, \operatorname{ArH}), 4.67-4.63$ $\left(1 \mathrm{H}, \mathrm{dd}, J=12.0,6.0 \mathrm{~Hz}, \mathrm{CHHNO}_{2}\right), 4.45-4.39\left(1 \mathrm{H}, \mathrm{m}, \mathrm{CHHNO}_{2}, 4.18-4.09\right.$

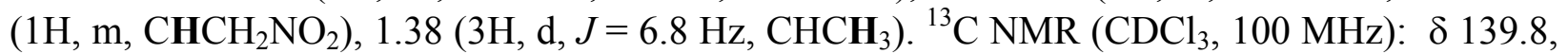
133.7, 129.1, 128.2, 127.4, 124.6, 80.3, 37.4, 17.8. HRMS Calcd. for $\mathrm{C}_{9} \mathrm{H}_{10} \mathrm{BrNO}_{2}$ : 242.9895 . Found: 242.9895. Anal. Calcd. for $\mathrm{C}_{9} \mathrm{H}_{10} \mathrm{BrNO}_{2}$ : C, 44.29; $\mathrm{H}, 4.13 ; \mathrm{N}, 5.74$. Found: C, 44.43; $\mathrm{H}, 4.97 ; \mathrm{N}, 5.57 .[\alpha]_{\mathrm{D}}{ }^{20}-2.83^{\circ}\left(c=1.60, \mathrm{CHCl}_{3}\right)$ for a sample that is $78 \%$ ee.

The optical purity of 1-nitro-2-(2-bromophenyl)propane is established by chiral GLC analysis (Chiraldex GTA, $140{ }^{\circ} \mathrm{C}, 15 \mathrm{psi}$ ) chromatograms are illustrated below for a $78 \%$ ee sample:
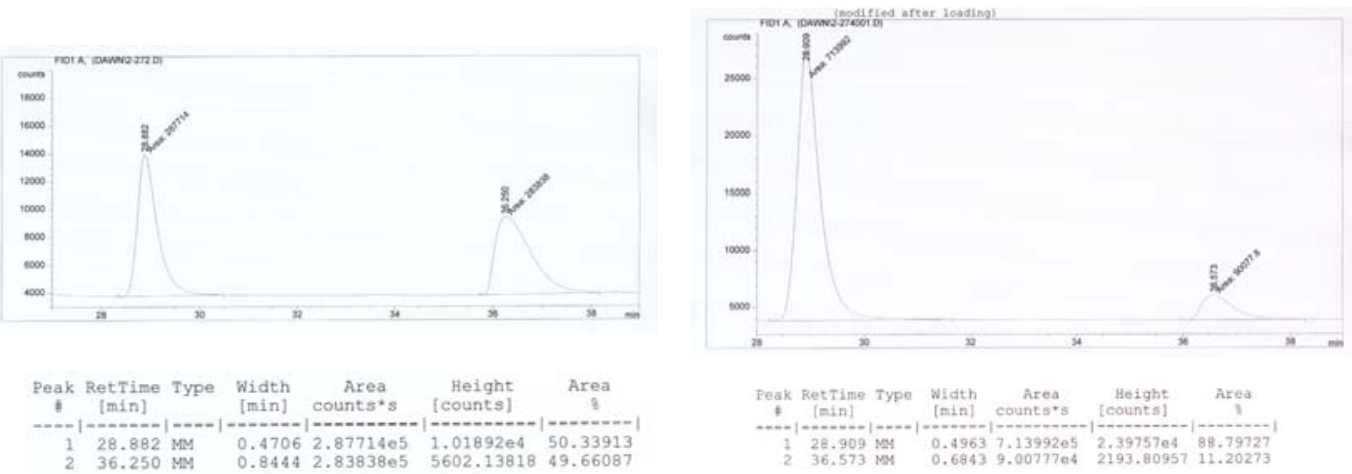

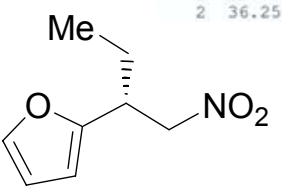

2q

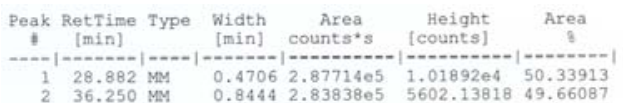

Nitro-2-(2-furyl)butane (2q). IR (neat): 3163 (w), 3119 (s), 2980 (s), 2943 (m), $2892(\mathrm{~m}), 1552$ (s), 1508 (m), $1464(\mathrm{~m}), 1438(\mathrm{~m}), 1382$ (s), $1350(\mathrm{w})$, $1193(\mathrm{w}), 1162(\mathrm{~m}), 1124(\mathrm{w}), 1080(\mathrm{w}), 1030(\mathrm{~s}), 973$ (w), $929(\mathrm{~m}), 885(\mathrm{w})$, $809(\mathrm{~m}), 759(\mathrm{~s}), 652(\mathrm{w}), 608(\mathrm{~m}) .{ }^{1} \mathrm{H} \mathrm{NMR}\left(\mathrm{CDCl}_{3}, 400 \mathrm{MHz}\right): \delta$ 7.34-7.33 (1H, m, ArH), 6.30-6.28 (1H, m, ArH), 6.14-6.12 (1H, m, ArH), 4.63-4.50 (2H, m, $\left.\mathrm{CH}_{2} \mathrm{NO}_{2}\right)$, 3.54-3.47 (1H, m, $\left.\mathrm{CHCH}_{2} \mathrm{NO}_{2}\right), 1.81-1.64\left(2 \mathrm{H}, \mathrm{m}, \mathrm{CH}_{2} \mathrm{CH}_{3}\right), 0.89\left(3 \mathrm{H}, \mathrm{t}, J=7.2 \mathrm{~Hz}, \mathrm{CH}_{2} \mathrm{CH}_{3}\right)$. ${ }^{13} \mathrm{C} \mathrm{NMR}\left(\mathrm{CDCl}_{3}, 100 \mathrm{MHz}\right): \delta 152.5,142.3,110.4,107.4,78.4,39.6,24.3,11.4$. Anal. Calcd. 
for $\mathrm{C}_{8} \mathrm{H}_{11} \mathrm{NO}_{3}$ : C, $56.80 ; \mathrm{H}, 6.55 ; \mathrm{N}, 8.28$. Found: $\mathrm{C}, 56.90 ; \mathrm{H}, 6.52 ; \mathrm{N}, 8.15 .[\alpha]_{\mathrm{D}}^{20}-28.6^{\circ}(c=$ $0.920, \mathrm{CHCl}_{3}$ ) for a sample that is $95 \%$ ee.

The optical purity of 1-Nitro-2-(2-furyl)butane is established by chiral GLC analysis ( $\beta$-dex, $120^{\circ} \mathrm{C}, 15 \mathrm{psi}$ ) chromatograms are illustrated below for a $95 \%$ ee sample:
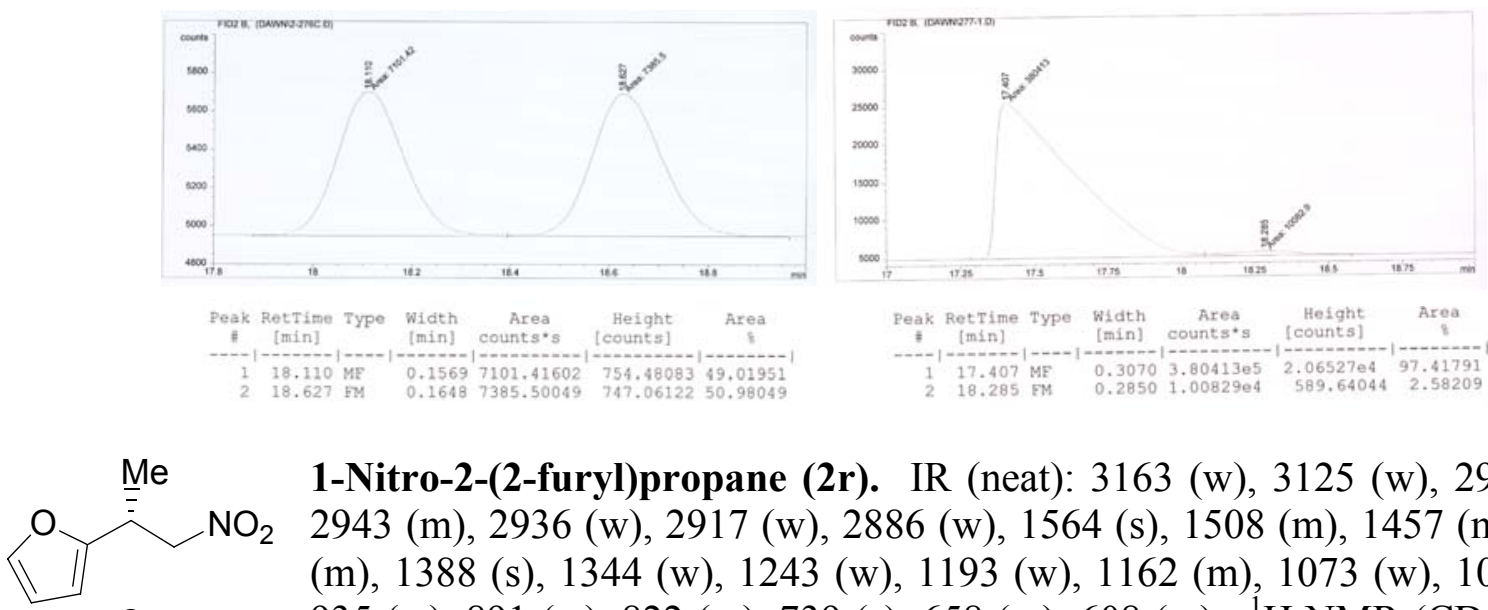

1-Nitro-2-(2-furyl)propane (2r). IR (neat): 3163 (w), 3125 (w), 2993 (m), 2943 (m), 2936 (w), $2917(\mathrm{w}), 2886$ (w), $1564(\mathrm{~s}), 1508$ (m), 1457 (m), 1438 (m), 1388 (s), $1344(\mathrm{w}), 1243$ (w), 1193 (w), $1162(\mathrm{~m}), 1073(\mathrm{w}), 1029(\mathrm{~m})$, 935 (w), 891 (w), $822(\mathrm{~m}), 739$ (s), $658(\mathrm{~m}), 608(\mathrm{~m}) .{ }^{1} \mathrm{H} \mathrm{NMR}\left(\mathrm{CDCl}_{3}, 400\right.$ $\mathrm{MHz}): \delta$ 7.34-7.33 (1H, m, ArH), 6.30-6.28 (1H, m, ArH), 6.12-6.11 (1H, m, ArH), 4.67-4.63 $\left(1 \mathrm{H}, \mathrm{dd}, J=12.0,6.4 \mathrm{~Hz}, \mathrm{CHHNO}_{2}\right), 4.44-4.39\left(1 \mathrm{H}, \mathrm{m}, \mathrm{CHHNO}_{2}\right), 3.77-3.68(1 \mathrm{H}, \mathrm{m}$, $\left.\mathrm{CHCH}_{2} \mathrm{NO}_{2}\right), 1.36\left(3 \mathrm{H}, \mathrm{d}, J=6.8 \mathrm{~Hz}, \mathrm{CHCH}_{3}\right) .{ }^{13} \mathrm{C} \mathrm{NMR}\left(\mathrm{CDCl}_{3}, 100 \mathrm{MHz}\right): \delta 154.0,142.3$, 110.4, 106.1, 79.7, 32.6, 16.3. Anal. Calcd. for $\mathrm{C}_{7} \mathrm{H}_{9} \mathrm{NO}_{3}: \mathrm{C}, 54.19 ; \mathrm{H}, 5.85 ; \mathrm{N}, 9.03$. Found: $\mathrm{C}$, $54.10 ; \mathrm{H}, 5.98 ; \mathrm{N}, 8.90 .[\alpha]_{\mathrm{D}}^{20}-21.0^{\circ}\left(c=1.04, \mathrm{CHCl}_{3}\right)$ for a sample that is $92 \%$ ee.

The optical purity of 1-Nitro-2-(2-furyl)propane is established by chiral GLC analysis ( $\beta$-dex, $100{ }^{\circ} \mathrm{C}, 15 \mathrm{psi}$ ) chromatograms are illustrated below for a $92 \%$ ee sample:
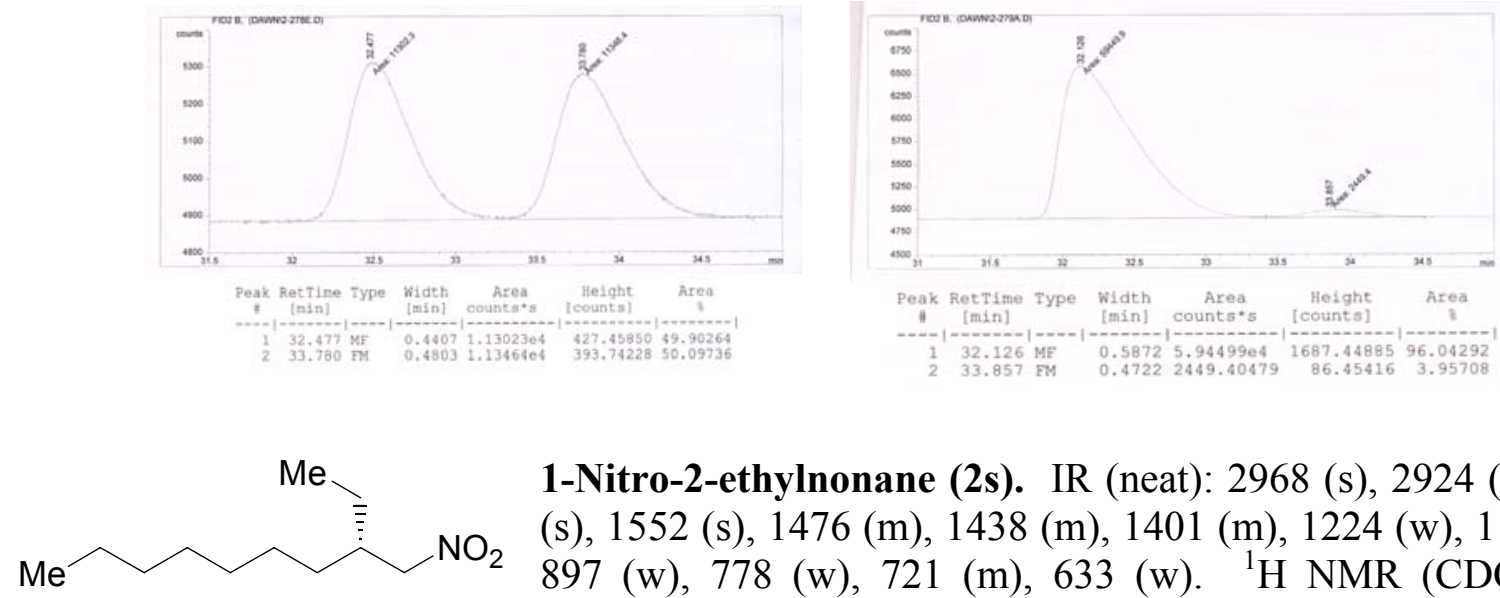

1-Nitro-2-ethylnonane (2s). IR (neat): 2968 (s), 2924 (s), 2867 (s), 1552 (s), $1476(\mathrm{~m}), 1438(\mathrm{~m}), 1401(\mathrm{~m}), 1224(\mathrm{w}), 1149(\mathrm{w})$, $897(\mathrm{w}), 778(\mathrm{w}), 721(\mathrm{~m}), 633(\mathrm{w}) .{ }^{1} \mathrm{H} \mathrm{NMR}\left(\mathrm{CDCl}_{3}, 400\right.$ 2s $\mathrm{MHz}): \delta 4.29\left(2 \mathrm{H}, \mathrm{d}, J=6.8 \mathrm{~Hz}, \mathrm{CH}_{2} \mathrm{NO}_{2}\right), 2.17-2.08(1 \mathrm{H}, \mathrm{m}$, $\left.\mathrm{CHCH}_{2} \mathrm{NO}_{2}\right), 1.28-1.26\left(14 \mathrm{H}, \mathrm{m}\right.$, aliphatic $\left.\mathrm{CH}_{2}\right), 0.91(3 \mathrm{H}, \mathrm{t}, J=$ $\left.7.2 \mathrm{~Hz}, \mathrm{CH}_{3}\right) 0.87\left(3 \mathrm{H}, \mathrm{t}, J=6.0 \mathrm{~Hz}, \mathrm{CH}_{3}\right) .{ }^{13} \mathrm{C} \mathrm{NMR}\left(\mathrm{CDCl}_{3}, 100 \mathrm{MHz}\right): \delta 79.5,39.0,31.9$, $30.9,29.8,29.9,26.4,24.1,22.3,14.2,10.6 .[\alpha]_{\mathrm{D}}{ }^{20}-2.20^{\circ}\left(c=0.667, \mathrm{CHCl}_{3}\right)$ for a sample that is $93 \%$ ee.

The optical purity of 1-nitro-2-ethylnonane is established by chiral GLC analysis ( $\beta$-dex, 90 ${ }^{\circ} \mathrm{C}, 20 \mathrm{psi}$ ); chromatograms are illustrated below for a $93 \%$ ee sample: 

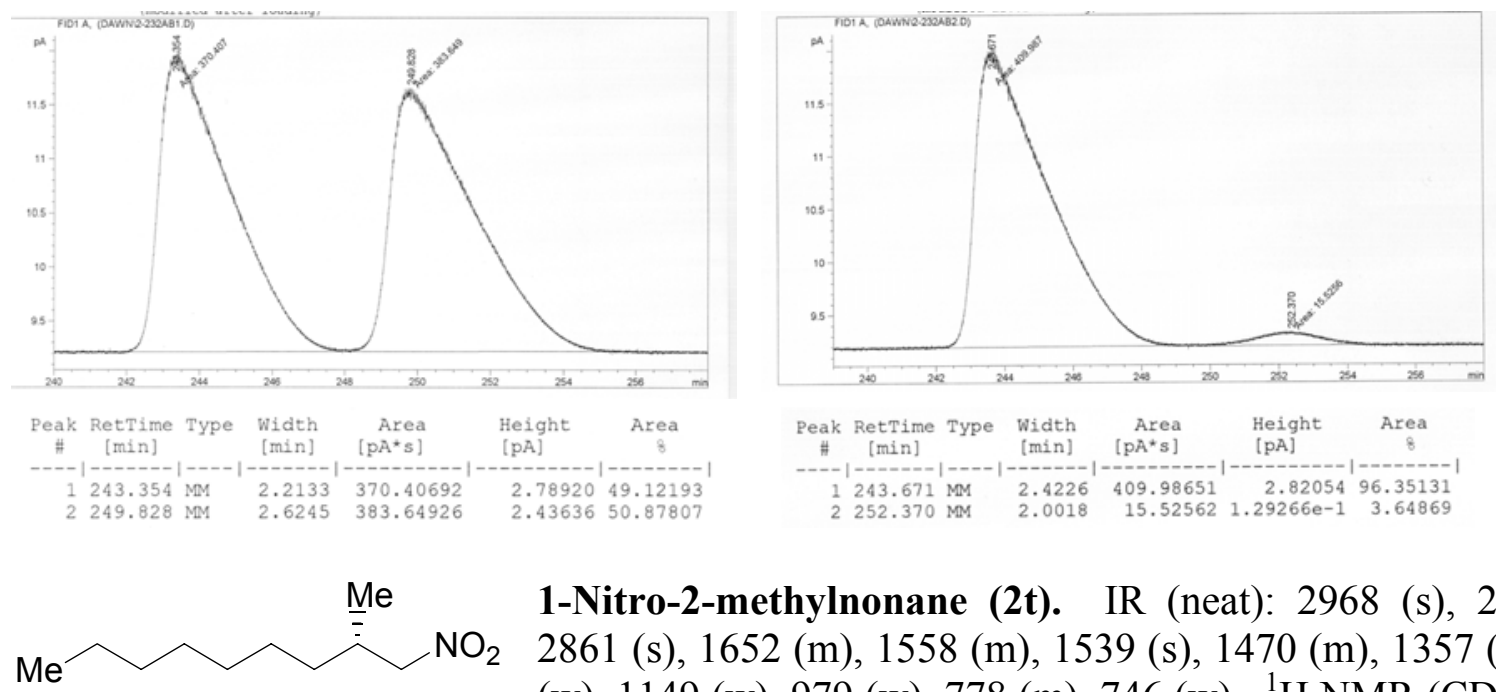

1-Nitro-2-methylnonane (2t). IR (neat): 2968 (s), 2936 (s), $2861(\mathrm{~s}), 1652(\mathrm{~m}), 1558(\mathrm{~m}), 1539(\mathrm{~s}), 1470(\mathrm{~m}), 1357(\mathrm{~s}), 1224$ (w), 1149 (w), $979(\mathrm{w}), 778(\mathrm{~m}), 746(\mathrm{w}) .{ }^{1} \mathrm{H} \mathrm{NMR}\left(\mathrm{CDCl}_{3}, 400\right.$ $2 \mathrm{t}$ $\mathrm{MHz}): \quad \delta 4.33-4.28\left(1 \mathrm{H}, \mathrm{m}, \mathrm{CHHNO}_{2}\right), 4.19-4.13(1 \mathrm{H}, \mathrm{m}$, $\left.\mathrm{CHHNO}_{2}\right), 2.34-2.25\left(1 \mathrm{H}, \mathrm{m}, \mathrm{CHCH}_{2} \mathrm{NO}_{2}\right), 1.36-1.26\left(12 \mathrm{H}, \mathrm{m}\right.$, aliphatic $\left.\mathrm{CH}_{2}\right), 1.01-0.98(3 \mathrm{H}$, $\left.\mathrm{d}, J=6.8 \mathrm{~Hz}, \mathrm{CH}_{3}\right) 0.87\left(3 \mathrm{H}, \mathrm{t}, J=6.4 \mathrm{~Hz}, \mathrm{CH}_{3}\right) .{ }^{13} \mathrm{C} \mathrm{NMR}\left(\mathrm{CDCl}_{3}, 100 \mathrm{MHz}\right): \delta 79.5,39.0$, 31.9, 30.9, 29.8, 26.4, 24.1, 22.3, 14.2, 10.6. HRMS Calcd. for $\mathrm{C}_{10} \mathrm{H}_{21} \mathrm{NO}_{2}$ : 188.1651. Found: 188.1620. Anal. Calcd. for $\mathrm{C}_{10} \mathrm{H}_{21} \mathrm{NO}_{2}$ : C, 64.13; H, 11.30; N, 7.48. Found: C, 64.38; H, 11.68; $\mathrm{N}, 7.21 .[\alpha]_{\mathrm{D}}{ }^{20}-1.41^{\circ}\left(c=0.993, \mathrm{CHCl}_{3}\right)$ for a sample that is $85 \%$ ee.

The optical purity of 1-nitro-2-methylnonane is established by chiral GLC analysis ( $\beta$-dex, 90 ${ }^{\circ} \mathrm{C}, 20 \mathrm{psi}$ ); chromatograms are illustrated below for a $85 \%$ ee sample:
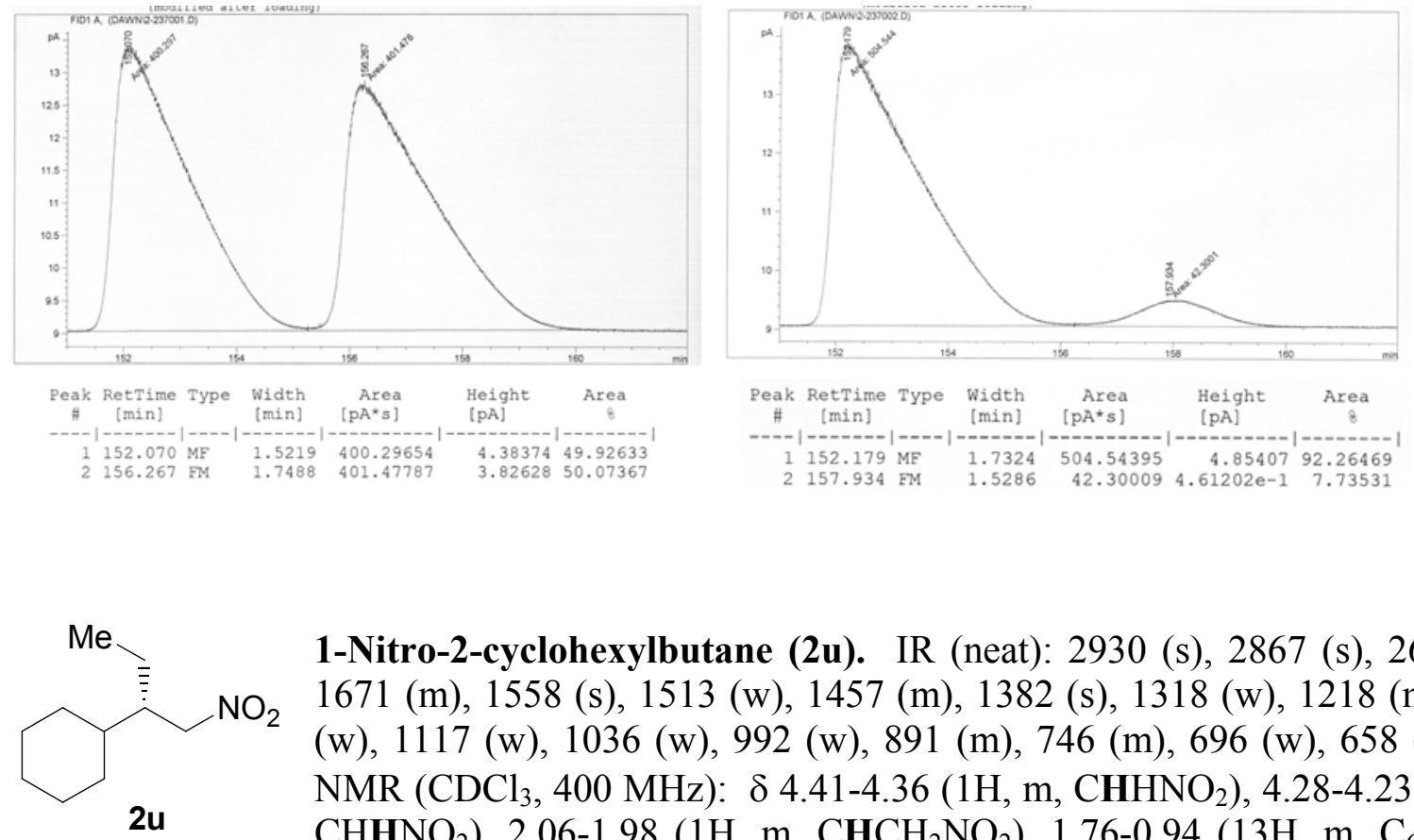

1-Nitro-2-cyclohexylbutane (2u). IR (neat): 2930 (s), 2867 (s), 2672 (w), $1671(\mathrm{~m}), 1558$ (s), 1513 (w), 1457 (m), 1382 (s), 1318 (w), $1218(\mathrm{~m}), 1142$ (w), $1117(\mathrm{w}), 1036(\mathrm{w}), 992(\mathrm{w}), 891(\mathrm{~m}), 746(\mathrm{~m}), 696(\mathrm{w}), 658(\mathrm{w}) .{ }^{1} \mathrm{H}$ $\mathrm{NMR}\left(\mathrm{CDCl}_{3}, 400 \mathrm{MHz}\right): \delta$ 4.41-4.36 (1H, m, CHHNO 2$), 4.28-4.23(1 \mathrm{H}, \mathrm{m}$, $\left.\mathrm{CHHNO}_{2}\right), 2.06-1.98\left(1 \mathrm{H}, \mathrm{m}, \mathrm{CHCH}_{2} \mathrm{NO}_{2}\right), 1.76-0.94\left(13 \mathrm{H}, \mathrm{m}, \mathrm{C}_{6} \mathrm{H}_{11}\right.$ and 


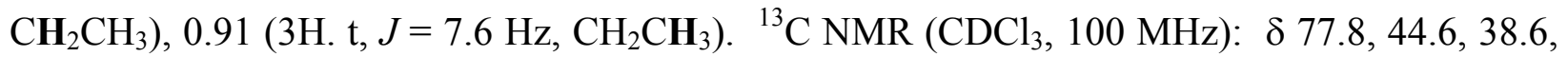
29.8, 29.5, 26.6, 21.6, 11.5. Anal. Calcd. for $\mathrm{C}_{10} \mathrm{H}_{19} \mathrm{NO}_{2}$ : C, 64.83; $\mathrm{H}, 10.34 ; \mathrm{N}, 7.56$. Found: $\mathrm{C}, 64.81 ; \mathrm{H}, 10.34 ; \mathrm{N}, 7.56 .[\alpha]_{\mathrm{D}}{ }^{20}-6.66^{\circ}\left(c=1.06, \mathrm{CHCl}_{3}\right)$ for a sample that is $95 \%$ ee.

The optical purity of 1-nitro-2-cyclohexylbutane is established by chiral GLC analysis (Chiraldex GTA, $80^{\circ} \mathrm{C}, 15 \mathrm{psi}$ ); chromatograms are illustrated below for a $95 \%$ ee sample:
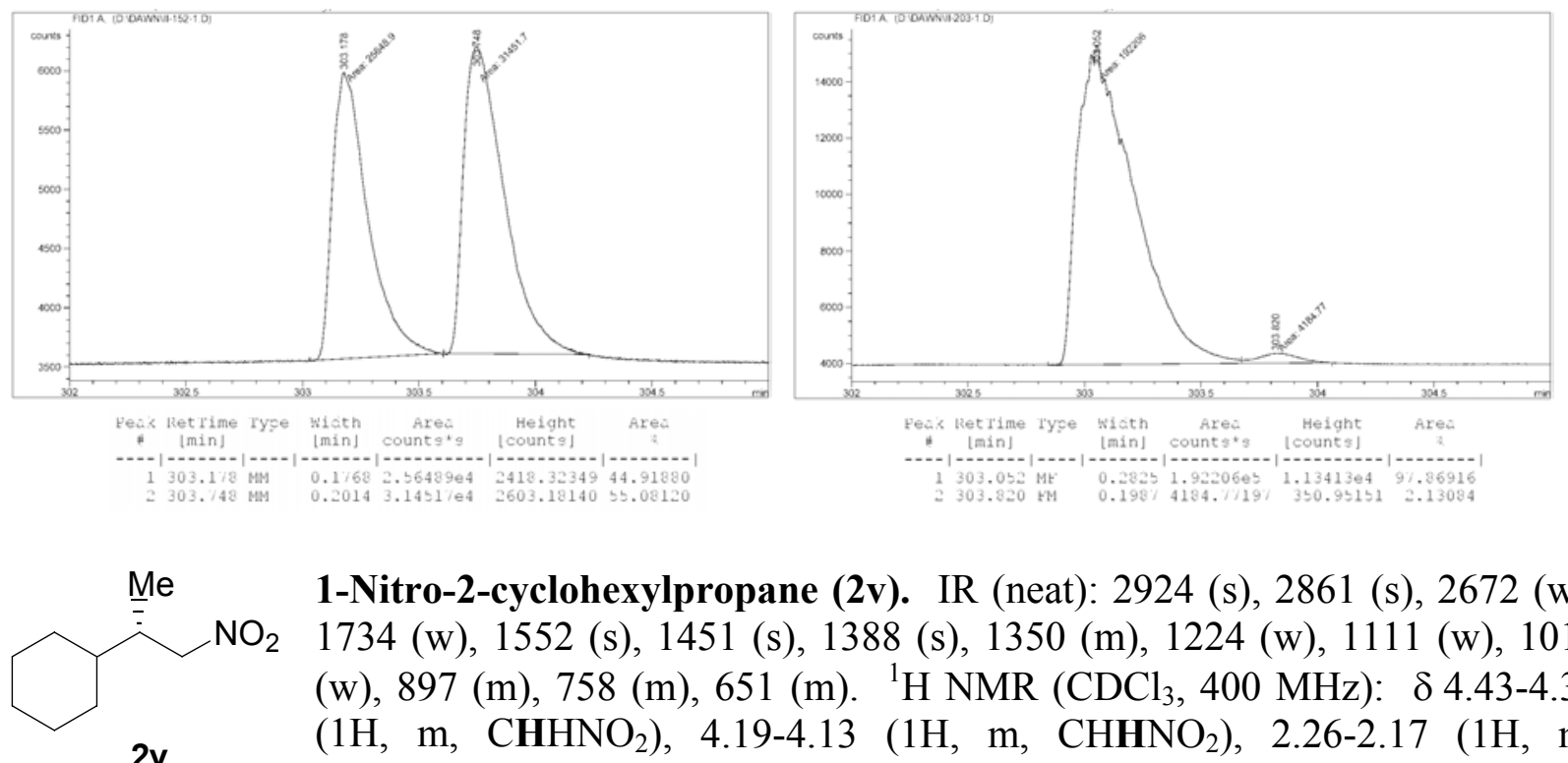

1-Nitro-2-cyclohexylpropane (2v). IR (neat): 2924 (s), 2861 (s), 2672 (w), 1734 (w), 1552 (s), 1451 (s), 1388 (s), 1350 (m), 1224 (w), $1111(\mathrm{w}), 1010$ (w), 897 (m), $758(\mathrm{~m}), 651(\mathrm{~m}) .{ }^{1} \mathrm{H}$ NMR $\left(\mathrm{CDCl}_{3}, 400 \mathrm{MHz}\right): \delta 4.43-4.38$ $\left(1 \mathrm{H}, \mathrm{m}, \mathrm{CHHNO}_{2}\right), 4.19-4.13\left(1 \mathrm{H}, \mathrm{m}, \mathrm{CHHNO}_{2}\right), 2.26-2.17(1 \mathrm{H}, \mathrm{m}$, $\left.\mathrm{CHCH}_{2} \mathrm{NO}_{2}\right), 1.76-0.98\left(11 \mathrm{H}, \mathrm{m}, \mathrm{C}_{6} \mathrm{H}_{11}\right), 0.95\left(3 \mathrm{H}, \mathrm{d}, J=7.2 \mathrm{~Hz}, \mathrm{CHCH}_{3}\right)$. ${ }^{13} \mathrm{C} \mathrm{NMR}\left(\mathrm{CDCl}_{3}, 100 \mathrm{MHz}\right): \delta 80.4,40.6,37.9,30.4,28.9,26.6,26.5,26.4,14.2$. Anal. Calcd. for $\mathrm{C}_{9} \mathrm{H}_{17} \mathrm{NO}_{2}: \mathrm{C}, 63.13 ; \mathrm{H}, 10.01 ; \mathrm{N}, 8.18$. Found: $\mathrm{C}, 63.15 ; \mathrm{H}, 9.92 ; \mathrm{N}, 8.19 .[\alpha]_{\mathrm{D}}{ }^{20}-17.6^{\circ}(c$ $=0.753, \mathrm{CHCl}_{3}$ ) for a sample that is $87 \%$ ee.

The optical purity 1-nitro-2-cyclohexylpropane is established by chiral GLC analysis ( $\beta$-dex, $120^{\circ} \mathrm{C}, 10 \mathrm{psi}$ ); chromatograms are illustrated below for an $87 \%$ ee sample:
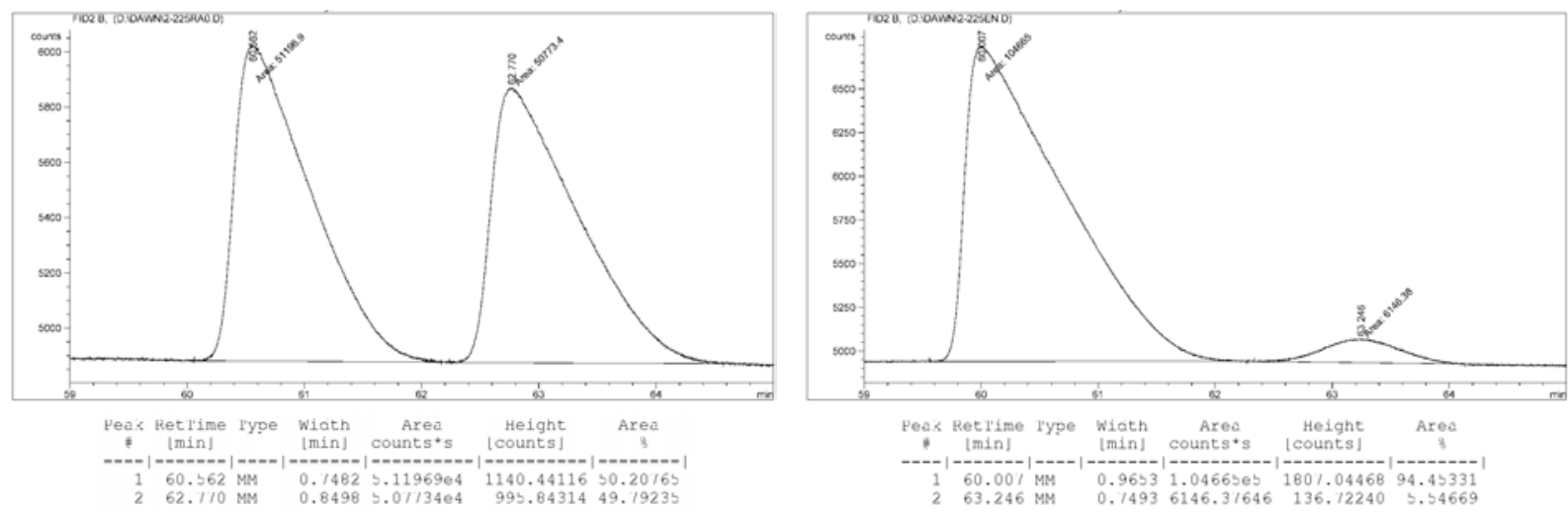


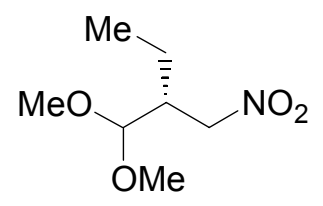

$2 \mathbf{w}$

(S)-1,1-Dimethoxy-2-ethyl-3-nitropropane (2w). IR (neat): 2980 (m), $2949(\mathrm{~m}), 2880(\mathrm{w}), 2829(\mathrm{~m}), 1552(\mathrm{~s}), 1457(\mathrm{~m}), 1388(\mathrm{~m}), 1212(\mathrm{w}), 1187$ (m), $1130(\mathrm{w}), 1080(\mathrm{~m}), 960(\mathrm{~m}), 778(\mathrm{w}), 620(\mathrm{w}) .{ }^{1} \mathrm{H}$ NMR $\left(\mathrm{CDCl}_{3}, 400\right.$ $\mathrm{MHz}): \delta 4.56-4.52\left(1 \mathrm{H}, \mathrm{dd}, J=13.2,6.0 \mathrm{~Hz}, \mathrm{CHHNO}_{2}\right), 4.32-4.27(1 \mathrm{H}, \mathrm{m}$, $\mathrm{CHHNO}_{2}$ and $\left.\left(\mathrm{OCH}_{3}\right)_{2} \mathrm{CH}\right), 3.40\left(3 \mathrm{H}, \mathrm{s}, \mathrm{OCH}_{3}\right), 3.38\left(3 \mathrm{H}, \mathrm{s}, \mathrm{OCH}_{3}\right), 2.51-$ $2.43\left(1 \mathrm{H}, \mathrm{m}, \mathrm{CHCH}_{2} \mathrm{NO}_{2}\right), 1.65-1.54\left(1 \mathrm{H}, \mathrm{dqd}, J=15.2,7.6,6.4 \mathrm{~Hz}, \mathrm{CHHCH}_{3}\right), 1.45-1.34(1 \mathrm{H}$,

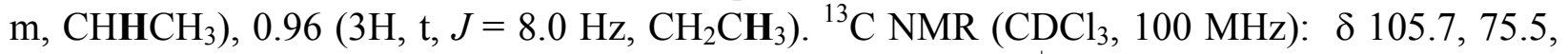
56.2, 55.0, 42.4, 20.9, 11.2. HRMS Calcd. for $\mathrm{C}_{7} \mathrm{H}_{15} \mathrm{NO}_{4}\left[\mathrm{M}-\mathrm{H}^{+}\right]$: 176.0923. Found: 176.0906. $[\alpha]_{\mathrm{D}}{ }^{20}+4.44^{\circ}\left(c=0.540, \mathrm{CHCl}_{3}\right)$ for a sample that is $84 \%$ ee.

The optical purity of 1,1-dimethoxy-2-ethyl-3-nitropropane is established by chiral GLC analysis (Chiraldex GTA, $120^{\circ} \mathrm{C}, 15$ psi) chromatograms are illustrated below for an $84 \%$ ee sample:
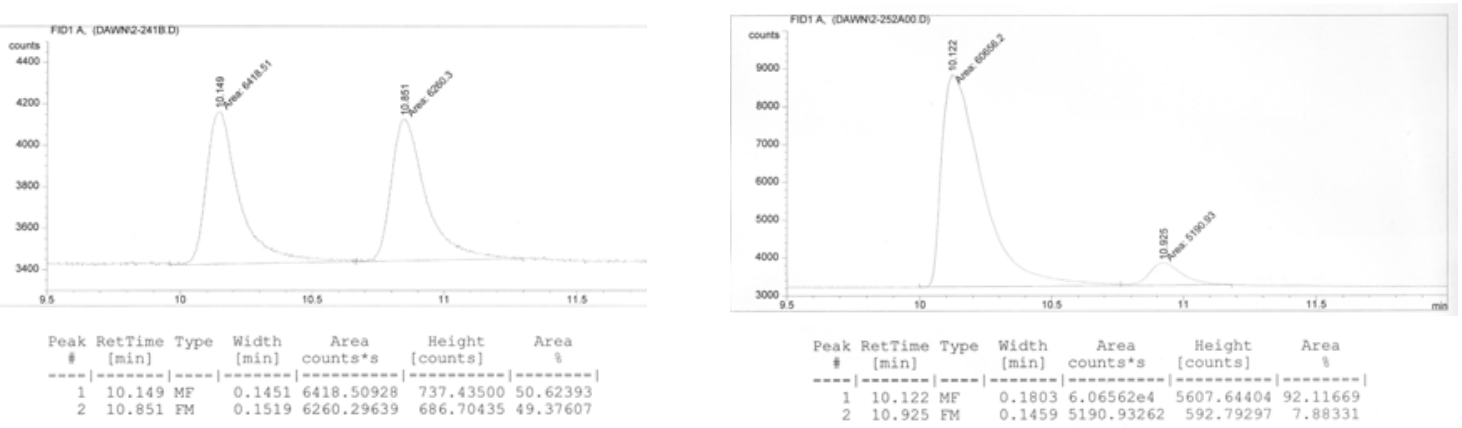

(S)-1,1-Dimethoxy-2-methyl-3-nitropropane (2x). IR (neat): 3113 (w), 2949 (m), 2848 (m), 1728 (w), 1564 (s), 1464 (m), 1382 (s), 1350 (s), 1281 (w), 1199 (m), $1130(\mathrm{w}), 1124$, (w), 1080 (s), 1004 (m), $954(\mathrm{~m}), 935(\mathrm{w})$, 853 (w), 765 (w), 734 (w), 608 (w), $551(\mathrm{w}) .{ }^{1} \mathrm{H} \mathrm{NMR}\left(\mathrm{CDCl}_{3}, 400 \mathrm{MHz}\right)$ : $\delta$ 4.56-4.51 $\left(1 \mathrm{H}, \mathrm{dd}, J=12.4,5.6 \mathrm{~Hz}, \mathrm{CHHNO}_{2}\right), 4.25-4.19(1 \mathrm{H}, \mathrm{m}$, $\mathrm{CHHNO}_{2}$ and $\left.\left(\mathrm{OCH}_{3}\right)_{2} \mathrm{CH}\right), 3.40\left(3 \mathrm{H}, \mathrm{s}, \mathrm{OCH}_{3}\right), 3.39\left(3 \mathrm{H}, \mathrm{s}, \mathrm{OCH}_{3}\right), 2.70-2.60(1 \mathrm{H}, \mathrm{m}$,

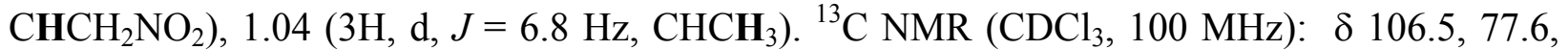
55.9, 54.7, 36.0, 13.0. HRMS Calcd. for $\mathrm{C}_{6} \mathrm{H}_{13} \mathrm{NO}_{4}[\mathrm{M}-\mathrm{H}]^{+}$: 162.0766. Found: 162.0761 . $[\alpha]_{\mathrm{D}}{ }^{20}+3.09^{\circ}\left(c=, \mathrm{CHCl}_{3}\right)$ for $77 \%$ ee product with $(S)$ stereochemistry. Literature value: $[\alpha]_{\mathrm{D}}^{21}-12.5^{\circ}\left(c=2.00, \mathrm{CHCl}_{3}\right)$ for $98 \%$ ee of $(R)$ product. ${ }^{6}$.

The optical purity of 1,1-dimethoxy-2-methyl-3-nitropropane is established by chiral GLC analysis (Chiraldex GTA, $80{ }^{\circ} \mathrm{C}, 20 \mathrm{psi}$ ); chromatograms are illustrated below for a $77 \%$ ee sample:

\footnotetext{
${ }^{6}$ Duursma, A.; Minnaard, A. J.; Feringa, B. L. J. Am. Chem. Soc. 2003, 125, 3700-3701.
} 

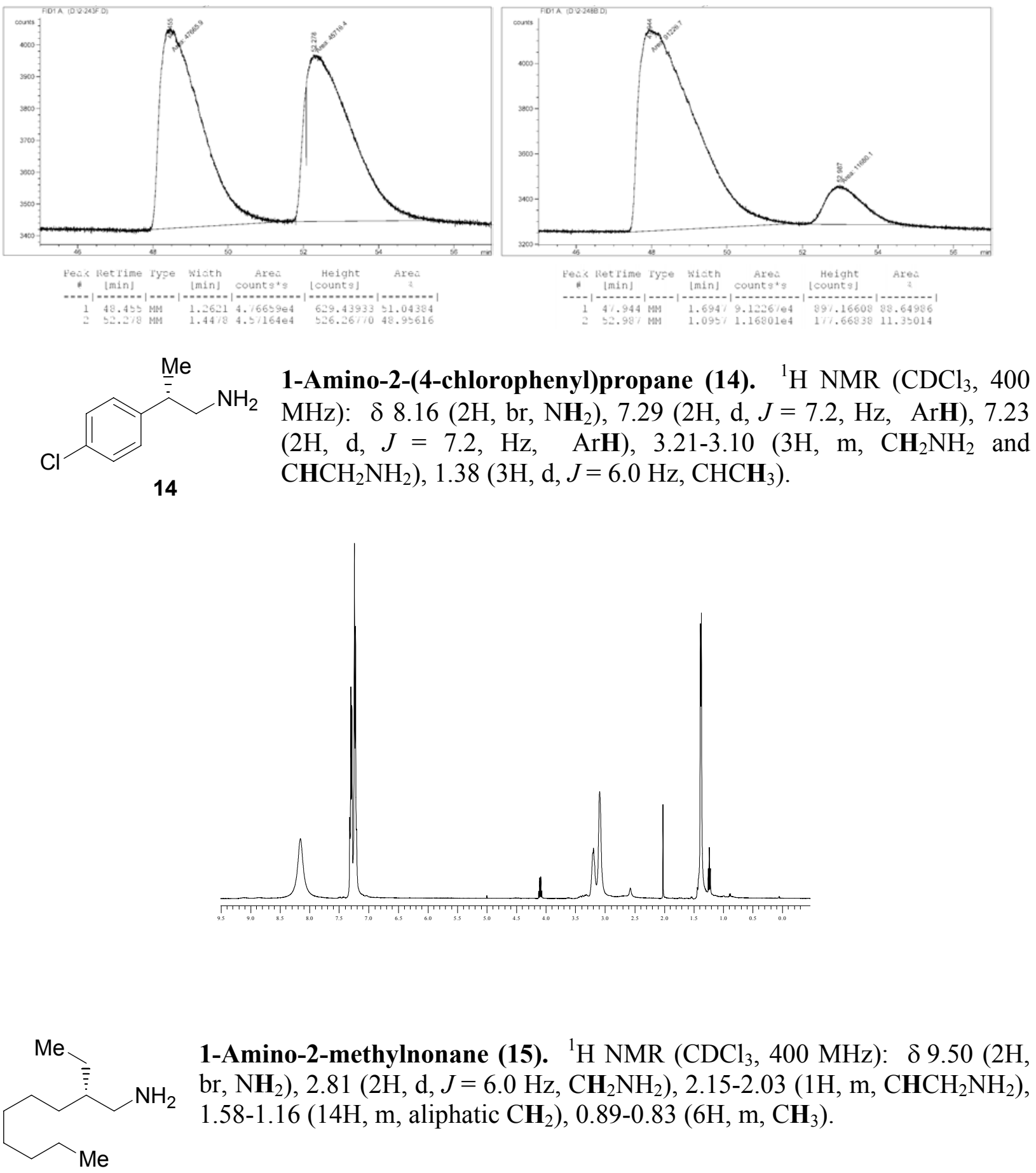

1-Amino-2-methylnonane (15). ${ }^{1} \mathrm{H}$ NMR $\left(\mathrm{CDCl}_{3}, 400 \mathrm{MHz}\right): \delta 9.50(2 \mathrm{H}$, br, $\left.\mathrm{NH}_{2}\right), 2.81\left(2 \mathrm{H}, \mathrm{d}, J=6.0 \mathrm{~Hz}, \mathrm{CH}_{2} \mathrm{NH}_{2}\right), 2.15-2.03\left(1 \mathrm{H}, \mathrm{m}, \mathrm{CHCH}_{2} \mathrm{NH}_{2}\right)$, 1.58-1.16 (14H, m, aliphatic $\left.\mathrm{CH}_{2}\right), 0.89-0.83\left(6 \mathrm{H}, \mathrm{m}, \mathrm{CH}_{3}\right)$.

15

Me 1-Amino-2-cyclohexylpropane (16). ${ }^{1} \mathrm{H} \mathrm{NMR}\left(\mathrm{CDCl}_{3}, 400 \mathrm{MHz}\right): \delta 2.69$ $\mathrm{NH}_{2} 2.64\left(1 \mathrm{H}, \mathrm{dd}, J=12.4,4.8 \mathrm{~Hz}, \mathrm{CHHNH}_{2}\right), 2.49-2.44(1 \mathrm{H}, \mathrm{dd}, J=12.8,7.6$ $\left.\mathrm{Hz}, \mathrm{CHHNH}_{2}\right), 1.72-0.88\left(14 \mathrm{H}, \mathrm{m}, \mathrm{CHCH}_{2} \mathrm{NH}_{2}, \mathrm{C}_{6} \mathbf{H}_{11}\right.$, and $\left.\mathrm{NH}_{2}\right), 0.83(3 \mathrm{H}$, 16 $\left.\mathrm{d}, J=6.8 \mathrm{~Hz}, \mathrm{CHCH}_{3}\right) .{ }^{13} \mathrm{C} \mathrm{NMR}\left(\mathrm{CDCl}_{3}, 100 \mathrm{MHz}\right): \delta 46.1,41.8,40.4$, $31.2,30.4,28.7,27.0,26.9,26.8,14.3$. 
Mampreian \& Hoveyda, Page S18
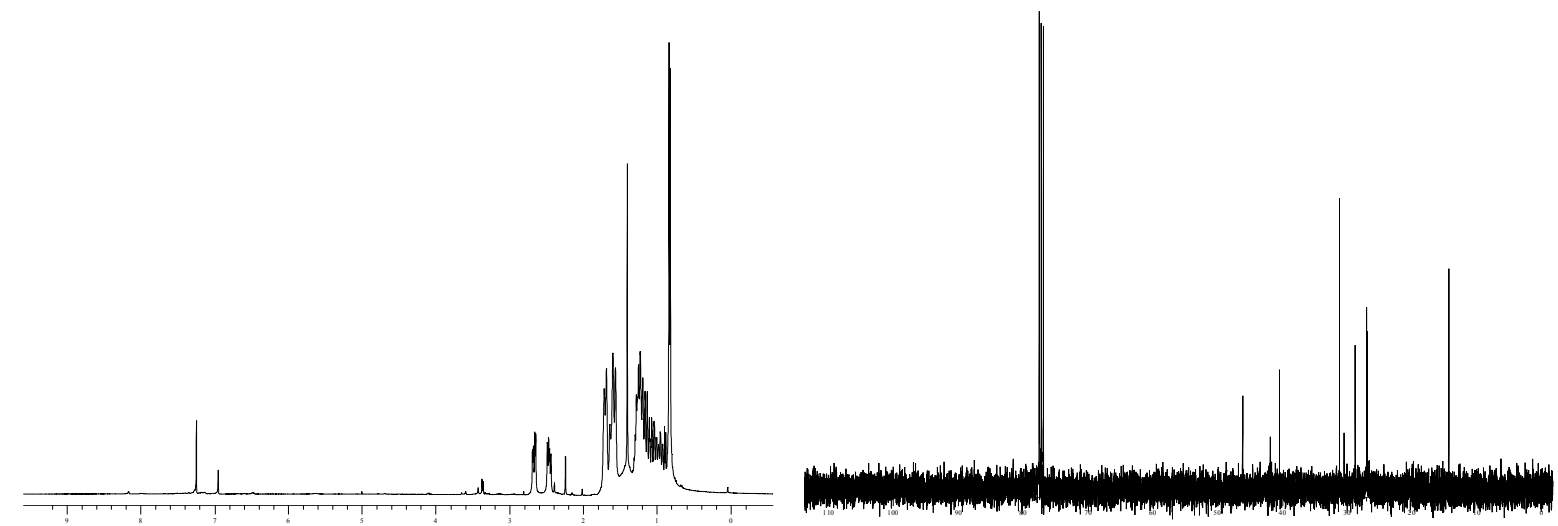